\title{
Energy-Pivotal Solutions for Green Femtocell Power Control in Hybrid-Dense Deployments
}

\author{
Mazen Al Haddad and Magdy Bayoumi \\ Center for Advanced Computer Studies, University of Louisiana \\ at Lafayette, LA, USA \\ E-mail: $\{$ mxa0001; mab0778\}@louisiana.edu
}

Received 17 June 2016; Accepted 7 September 2016;

Publication 22 September 2016

\begin{abstract}
With the ever-increasing demand for mobile traffic, it is important that telecom networks are modernized with all the capacity, quality and coverage extension technologies available. Due to the pressures of population and modern technology, the biophysical environment is being degraded, sometimes permanently. Energy efficiency and low carbon strategies have recently attracted a lot of concern. Solutions that help reduce greenhouse gases and make a less harmful environmental impact are required. Heterogeneous Networks (HetNets), comprising a mix of lower-power nodes (like Femtocells) and higher power Macro base-stations, provide the means to enhance quality and coverage and offer scalable networks towards 1,000 fold gains in capacity as per 5G technology vision. Femtocells increase the capacity of the Macro-only networks by moving high-data rate users to low-power Femto alternatives and are likely to proliferate in next-generation wireless systems. High-density Femtocell deployments are seen as future low-cost solution to significantly gain capacity compared to conventional deployments. However, in such deployments, the problem of interference appears between the Macrocells and Femtocells as well as among the Femtocells themselves. Expensive insights into Macro-Femto interference are researched and available, while Femto-Femto interference is only partially addressed. This research work
\end{abstract}

Journal of Green Engineering, Vol. 6, 99-144.

doi: 10.13052/jge1904-4720.615

(c) 2016 River Publishers. All rights reserved. 
outlines this problem and points to possible solutions to mitigate FemtoFemto interference, increase network capacity, decrease power consumption and likewise reduce the $\mathrm{CO}_{2}$ footprint.

Keywords: Femtocell, HetNets, Interference Management, RES, LTE, $\mathrm{CO}_{2}$ Emission, Power Saving.

\section{Introduction}

The strong demand for mobile data is obvious and will continue to grow exponentially in the next decade. The associated significant impact on global warming and greenhouse gas emissions has been also observed. Existing telecommunication radio technology like the Long-Term Evolution (LTE) network is reaching the limits of available spectrum and network capacity for mobile data applications (Shannon's law). The lack of radio spectrum due to more and more requests for bandwidth by end users in order to achieve high transmission rates and high levels of Quality of Service (QoS) is a major unsolved issue. More than $50 \%$ of all voice calls and $70 \%$ of data traffic originates indoors [1], where, for indoor users, propagation and penetration losses make high signal quality and data rates very challenging to realise.

Cisco Visual Networking Index (VNI) Global Mobile Data Traffic Forecast shows major global mobile data traffic projections and growth trends [2]. Overall mobile data traffic is expected to grow to nearly a 10-fold increase by 2019 over 2014. Mobile data traffic will grow at a CAGR of 57 percent from 2014 to 2019 (Figure 1).

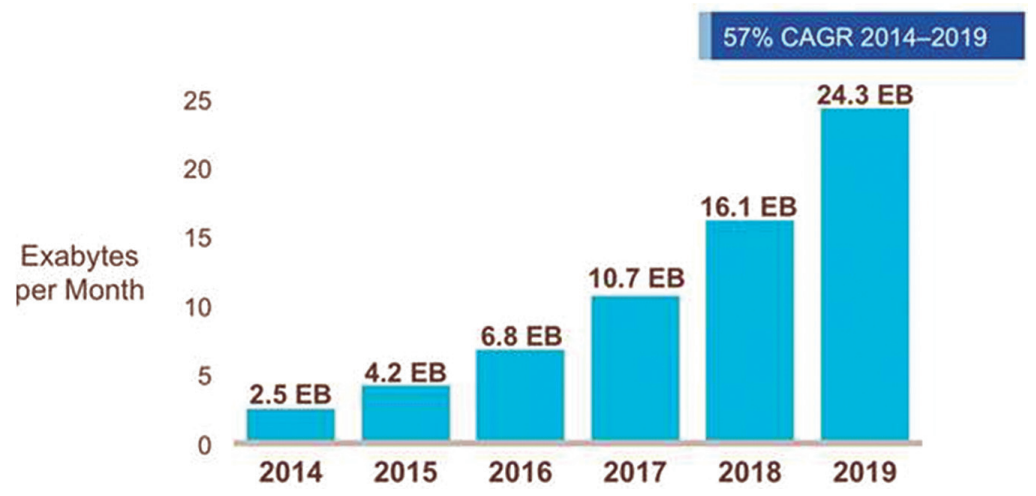

Figure 1 Cisco Forecasts 24.3 Exabytes per Month of Mobile Data Traffic by 2019. 
Nokia Networks Technology Vision 2020 for future mobile networks supports up to 1,000 times more capacity [3]. Projections, using a figure of 75 percent average year-on-year global growth, show that by 2020 traffic will increase to 1,000 times more than 2010 levels. It is clear that networks need to be prepared to handle this level of growth in order to fully monetize mobile broadband demand. The only viable way of reaching "the 1000×" paradigm is to make cells smaller, denser and smarter. As a result, dense deployments of small cells are crucial in addressing the $1000 \times$ challenge. According to SMALL CELL FORUM, which drives the wide-scale adoption of small cells and accelerate the delivery of integrated HetNets, a distribution of $85 \%$ small cells and $15 \%$ macro is expected by 2020 .

Therefore, it is time for new techniques to overcome the current capacity and link budget limitations while at the same time minding the impact on the environment. Current candidate technologies are 5G and LTE next advanced generation where heterogeneous networks with dense Femtocell grids are identified as potential areas for capacity extensions and improvements.

Mobile networks, and especially their radio access parts (frontend), are by far the dominant and most concerning drain on energy in the entire network [4] and thus an enormous introduction of global $\mathrm{CO}_{2}$ emissions. The findings of researchers from the University of Melbourne's partner Centre for Energy Efficient Telecommunications, and Bell Labs, show that the Information and Communications Technology (ICT) industry is guilty of producing more than 830 million tons of carbon dioxide $\left(\mathrm{CO}_{2}\right)$ annually, which is about 2 per cent of global $\mathrm{CO}_{2}$ emissions, and is expected to double by 2020 [5].

As exposed in Figure 2, the energy calculations show up to $90 \%$ of this consumption is attributable to mobile and access networks (data centres account for only $9 \%$ ).

Femtocell deployments are seen as good cost-efficient solutions for providing higher capacity and coverage for indoor network environments, in addition to required capacity increase and high data rates or qualified coverage guarantee in hard-to-cover areas. They plug the coverage holes (a.k.a. dead zones) and ideally complement Macro networks with the transmitter closer to user. System efficiency is then much higher with a guarantee of sufficient high bandwidth and mobile coverage, where Macro fails. Typical application areas of dense Femtocell deployments are dense urban residential regions, mall/enterprise places, stadiums/venues, downtown, business regions, etc.

Femtocells or Home NodeBs have been a hot topic for quite some time since they offer the end-user better signal quality, more reliable communications and higher throughputs, as well as power and battery savings due to 


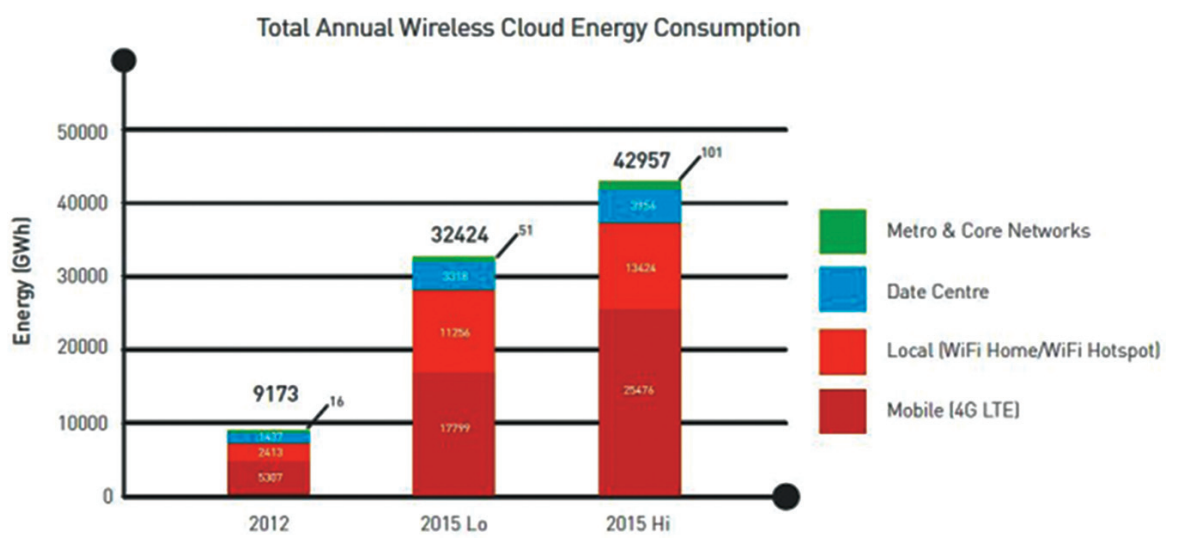

Figure 2 Estimate for annual energy consumption broken down into the various components of the wireless ecosystem, 2012 and 2015 (Lo and Hi scenarios).

the reduced distance between the transmitter and the receiver [6]. Femtocells will also help to manage the exponential growth of the traffic, thanks to the handover of the indoor traffic to the backhaul.

In the overlaid Macrocell coverage area Femtocell-to-Macrocell, Femtocell-to-Femtocell and Macrocell-to-Femtocell interferences may occur. The frequency of these interferences increases as the density of Femtocells is increased. In a dense Femtocell network deployment, thousands of Femtocells can be deployed within a small coverage area and thus the system must be able to manage efficiently [7]. Of course, dense and unplanned deployments may result in harmful co-channel interference. Therefore, Femto coverage should be limited to the intended deployment area only. Spill-over into the Macro or neighbouring Femto coverage areas will cause interference in these domains and without proper interference management, significant power is likely to be wasted in order to maintain acceptable user performance and QoS.

Higher Femtocell transmit power can provide wider coverage and better own service quality but it may also cause tremendous interference to other surrounding Femtocells and users of the adjacent Macro and Femtocells. Proper setting of the Femtocell transmit power level will help manage the interand intra-interference, while maintaining good Femtocell performance and increasing the network capacity. In order to successfully react to the changes of the traffic and channel, and minimize interference in femtocell deployments, the use of sophisticated self-organization techniques is needed. Selforganization will allow femtocells to integrate themselves into the network of 
the operator, learn about their environment (neighbouring cells, interference) and tune their parameters (like power and frequency) accordingly.

In this work we focus on the Femto interference aspects especially in massive deployments. In the light of most known facts about Femtocell technology, the major goal of this work is to outline the Femto-to-Femto interference problem, which is as yet only partially addressed, and provide comprehensive, self-organising and environmentally friendly power control frameworks with special focus on dense Femtocell deployments.

The remainder of the paper is organized as follows. In Section 2 we briefly discuss the challenges of Femtocell deployments. In Section 3 we discuss the interference management techniques for downlink \& uplink power control and their current related work. In Section 4 we explain intra-interference management (between Femtocells). In Section 5 we present our proposed green power control frameworks, and their achieved performance and green impact are evaluated and discussed in Sections 6 and 7. Finally, conclusive remarks can be found in Section 8.

\section{Challenges of Femtocell Deployments}

A noteworthy amount of studies conclude that Femtocell technology is one of the most promising enablers of energy efficiency. The study in [8] indicates that, compared to a standard Macrocell deployment, Femtocell deployments may significantly reduce the energy consumption of both the access network and the mobile terminals from four to eight orders of magnitude.

In Heterogeneous Networks, Femtocells are deployed with Macrocells in an overlay, overlapping or disjointed area in cellular networks. With such hybrid-dense networks, the problem of interference comes between the Macro and small cells as well as among the small cells themselves. The interference between Macrocell and Femtocell, i.e., inter-tier interference, arises from the fact that Femtocells may utilize the spectrum already allocated to the Macrocell or other Femtocells. Without proper interference management, significant power is likely to be wasted in order to maintain acceptable user performance. Some other challenges to be considered with Femto technology are:

- Tx Power Management: each Femtocell needs to adapt its transmit power as other cells topology changes [9].

- Location Uncertainty: the location of the Femtocell is random and unpredictable, and as the owner likes [10]. Thus, convergence under 
time varying and unpredictable environmental changes is an essential requirement.

- Configuration Variation: the degree of uncertainty in the deployment increases if the Femtocells' configuration could be set differently for each Femtocell [10].

- Access and Security Control: OSG (Open Subscriber Group) or CSG (Closed Subscriber Group). Different access control mechanisms for Femtocells may result in different interference environments that are much more complicated to control than those of conventional wireless cellular networks [10].

- Resource Management: Femtocells deployed in co-channel manner as Macrocells need to coordinate with Macrocells to determine the optimal resource partitioning and maximize traffic offloading to Femtocells [11].

- Mobility Management: Femtocells need to discover neighbors autonomously to facilitate UE handover. Conventional handover schemes do not assure an optimal management of the handover procedures over the Heterogeneous Networks [12].

- No change of Macrocell RRM (Radio Resource Management): changing the Macrocell RRM should be avoided as it may influence alreadystabilized Macrocell user services and accompany costly optimizations.

- Backhaul Management: when Femtocells experience limitation in backhaul bandwidth, they should prioritize user classes or transferred packets [13].

- Equipment Compatibility: like support of legacy user devices which is an important requirement for market penetration.

All in all, introducing Femtocells should not significantly degrade the performance of other or prior deployed networks; therefore all the above challenges should be considered and solved. The main challenges of Femtocell technology are the coordination between Macrocells and ad-hoc Femtocells, interference mitigation with Macrocells and power-aware and green Femtocell deployment. Our research work tries to solve these challenges and focuses especially on dense deployments where these issues are currently present.

\section{Preliminaries and Interference Management Techniques}

It is crucial to mitigate the interference which arises when Femtocells are deployed in Macrocell networks and ensure that the spectral efficiency is better than that of the Macrocell-only networks. In [6], it is shown that the throughput can be dramatically improved after introducing Femtocells to support the overloaded Macrocells but with proper interference mitigation techniques. 


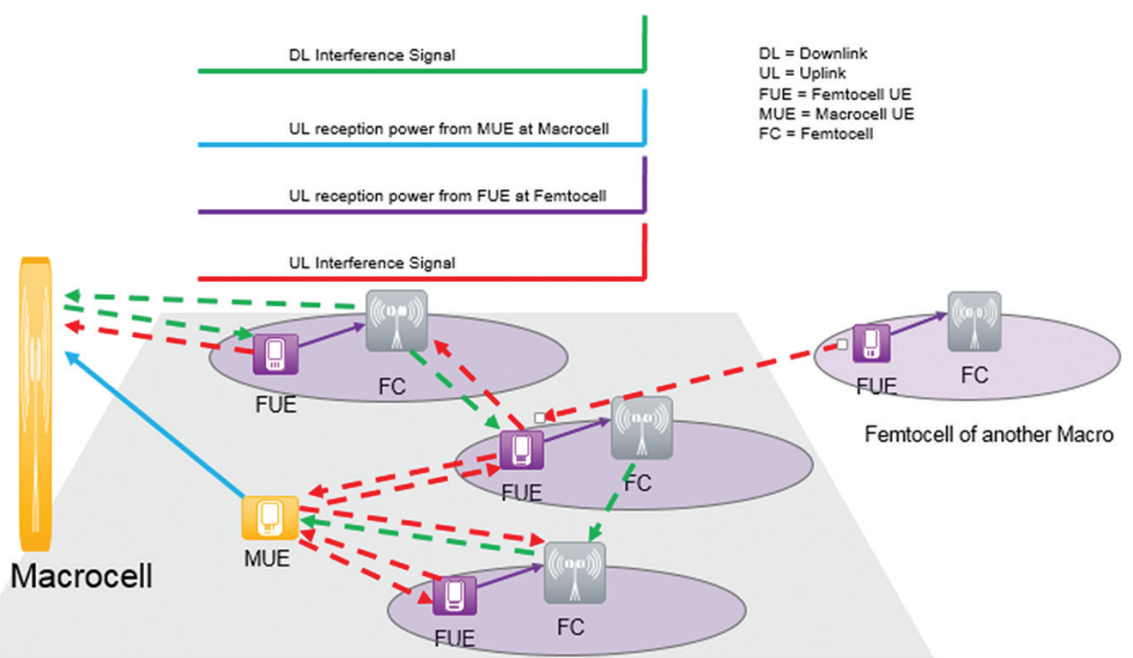

Figure 3 Typical interference in two-tier Macro-Femto network deployment.

Figure 3 shows some typical interference types in two-tier Macro-Femto networks. As the mass of deployed Femtocells increases, the rate of these interferences increases too.

Typical types of interference are:

- Downlink (DL) interference from the Femtocell to nearby Macro \& Femto UE (MUE \& FUE). A MUE far from its Macrocell will be affected the most.

- DL interference from nearby Macrocell to Femto UE (it can lower the SINR of FUE).

- DL interference among nearby Femtocell networks.

- Uplink (UL) interference from nearby MUE to Femtocell.

- UL interference from FUE to nearby Macrocell. Many active FUEs can cause severe interference to the Macrocell.

- UL interference among nearby Femtocell networks.

- UL interference from nearby FUE to neighbouring UEs of another Macrocell cluster; higher probability in dense deployments.

Several interference management techniques for heterogeneous cellular networks are possible:

1. Optimization of resource allocation in case of coexisting Femtocell and Macrocell networks (cognitive radio) as presented in [14], where selforganizing approaches for frequency assignment of OFDMA Femtocells 
are introduced, or by utilizing new features like in [15], where "cell range expansion" is showcased that allows User Equipment (UE) to be served by a cell with weaker received power.

2. Radio resource coordination by allocating different resources between neighbouring eNBs in the Time or Frequency domains as shown in [16].

3. System \& Design improvement by adding more resources like MIMO design as shown in [17].

4. Dynamic Resource Management where new techniques like "opportunistic small cells" are introduced [18], which dynamically turns the Femtocell "ON" or "OFF" based on the need for capacity, for instance proximity of users or traffic status e.g., idle status, to not only reduce interference but also lower energy consumption.

5. Power Control (PC) which is necessary to mitigate the interference by manipulating the transmission power settings.

In our work we mainly focus on the power control approach to mitigate different interference types with special preoccupation on carbon reduction and impact of environment. There are downlink and uplink power control techniques for interference mitigation and power setting tuning. In this section we try to summarize the most recent and important PC-related solutions and their related work.

\subsection{Uplink Power Control}

The uplink power control for LTE networks is defined according to current $3 \mathrm{GPP}$ standards and is composed of open and closed loop components which are referred to as OLPC and CLPC respectively. The transmit power setting of Physical Uplink Shared Channel (PUSCH) as in 3GPP is defined as follows (dB scale) [19]:

$$
\mathrm{P}=\min \left\{\mathrm{P}_{\max }, 10 \log _{10}(\mathrm{M})+\mathrm{P}_{0}+\alpha \cdot \mathrm{PL}+\Delta \mathrm{TF}(\mathrm{i})+\mathrm{f}(\mathrm{i})\right\}
$$

Where $\mathrm{P}_{\max }$ is the maximum allowed UE transmit power, $\mathrm{M}$ is the number of Physical Resource Blocks (PRB) scheduled for the given user in a time slot (bandwidth of the PUSCH resource assignment), $\mathrm{P}_{0}[\mathrm{dBm}]$ is target mean received power (user or cell specific), $\alpha$ is pathloss compensation factor (cell specific), $\mathrm{PL}[\mathrm{dB}]$ is the downlink pathloss measured by the UE, $\Delta \mathrm{TF}(\mathrm{i})[\mathrm{dB}]$ is a parameter that depends on the used Modulation and Coding Schema (user specific), and $\mathrm{f}(\mathrm{i})[\mathrm{dB}]$ is a user specific CLPC correction parameter. In this case the cell specific parameters of the OLPC are considered $\left(\mathrm{P}_{0}, \alpha\right)$ as they have the main impact on the inter-cell interference. 
If this basic power control method is applied in the uplink, a too strong signal transmitted from the outdoor Femtocell UE can possibly cause interference to nearby Macrocell(s). In order to deal with this problem, the method has to be extended and factors like PL between Femto UE and its nearest neighbour Macrocell need to be considered for additional actions.

Most known methods developed for uplink power control are:

Power Cap Based PC: the maximum transmission power density (i.e., power cap) of the Femtocell UE (FUE) is restricted in order to avoid heavy interference to macro eNB(s) [20].

PLdifference based PC: with knowledge of the difference between the PL from the Femto UE to its serving Home eNB and its nearest neighbour Macrocell, the Femtocell calculates the power offset as a non-decreasing function of the PL difference [20].

Adaptive target mean received power (adaptive $P_{0}$ ): $\mathrm{P}_{0}$ IS DEFINED in a way to reflect the distribution of interference levels within Macrocell, e.g., as a function of pathloss towards closest Macro eNB [21].

Link budget Analysis: the radio link quality for a cellular user is determined, given a set of $\mathrm{N}$ transmitting Femtocells with different SINR targets. Achieving higher SINR targets in one tier fundamentally constricts the highest SINRs obtainable in the other tier [22].

Distributed power control loops based: in [23] CTRL (Complementary TRicontrol Loop) controls the maximum TX power of Femtocell users, determines the target SINRs of Femtocell users and protects the femtocell users' uplink communications.

All the above mentioned approaches show a strong protection of Macrocell \& Femtocell users' uplink communications but don't consider all the surrounding conditions and particularly neighbouring Femtocells in dense and non-dense deployment scenarios.

Penalty Algorithm for LTE Uplink in Heterogeneous Networks: the Femto UE's $P_{0}$ is determined such that two UEs next to each other at the cell border between the macro and Femto cells, but with one attached to Macrocell and the other attached to the Femtocell should have the same transmit power per 
PRB [24]. This is called the "equal power at cell edge" setting. The issue with this method is that the power and interference level is set high for Edge Femto UEs.

Per-layer power settings based on the maximization of selected KPIs: UL PC settings improve the balance of UE throughput between layers, maximizing some selected KPIs [24]. KPI values need to be calculated for all UEs in the cell area (served by both Macro and Femto cells). The issue with this schema is that it's not flexible to RF surrounding changes. Only fixed parameter values are provided based on the testing approach.

Best chosen $P_{0}$ and Alpha values: by simulation the influence of $P_{0}$ for a fixed value of $\alpha=0.6$ in [25] and $\alpha=0.8$ in [26] it is shown that closed loop power control combined with FPC can greatly improve the performance achieved by a purely open loop scheme. In [27], the authors propose an analytical approach to derive an approximate equation for SNIR that is on the half-distance between cell and UEs. The issue with theses schemas is the high dependence on the path-loss coefficient only and the fixed $\alpha$ value used.

Furthermore, in many cases the parameters of UL power control $[20,21,24]$, such as the maximum allowed UE transmit power or the target received power (user or cell specific), are optimized on the basis of the path loss from the Femtocell base station or Femtocell UE to the Macrocell base station. The Path Loss is estimated by the difference between the transmit power of the UE and the received power at the base station. However, in this path loss based method, only the ULinterference of Femtocell UE to Macrocell is considered. Therefore, as the number of neighbouring Femtocells increases, the UL interference of Femtocell UEs is increased. As a result, the UL throughput degrades and UL UE transmit power goes up to compromise the increased interference. UL interference from Femtocell UE to other/neighbouring Femtocells and Femtocell UEs is not yet properly considered in existing Femtocell NW power control algorithms.

According to the above analysis, conventional and baseline fractional PC methods originally designed for heterogeneous networks are no longer suitable. To mitigate UL interference, it is important for Femtocells to control UL transmit power adaptively for the various transmission states of each Femtocell, such as its location in a Macrocell and the traffic load. In our work an UL power control method for LTE Femtocell UEs is proposed to adaptively find out the proper target received power (taking several RF circumstances into consideration) and set the UE transmit power accordingly. 


\subsection{Downlink Power Control}

The transmit power of Femtocell consists of pilot power which is responsible for cell and channel selection and traffic power including singling and data power. Large pilot power will result in large cell coverage and small pilot power may lead to insufficient coverage. Moreover, the larger the pilot power the less the power left for traffic, which results in minimizing the throughput of the Femtocell. In addition, large pilot power may introduce high outage probability to neighbouring non-CSG users due to interference [28].

Different interference mitigation approaches have been proposed in literature to properly control and set the Femtocell transmission power in DL. According to [29] power control techniques are classified based on different criteria such as non-assisted vs. assisted and centralized vs. distributed techniques. The non-assisted techniques set the Femtocell transmission power according to self-measurement reports or predetermined system parameters, whereas the assisted techniques are based on measurement reports from Macrocell UE (MUE) and Femtocell UE (FUE) or the coordination of the Femtocell with a Macrocell or neighbouring Femtocells.

The benefit of the non-assisted techniques is that no extensive signalling is required between Femtocell and Macrocell, FUE or MUE. However, there may be a significant difference between the RF conditions measured by Femtocell and those experienced by MUEs or FUEs. On the other hand, the assisted techniques can only be adopted if the Femtocell can successfully receive measurement reports from FUE and MUE or coordinate with the Macrocell and neighbouring Femtocells. Therefore, the assisted and nonassisted techniques can be applied in different operational scenarios, hence Femtocells should have a hybrid power setting scheme and switch between power setting modes according to the operation scenario.

Furthermore, the centralized power control techniques need a central controller and require extensive control signalling in the network, whereas the distributed techniques can avoid that bottleneck and could improve their reliability by eliminating the central entity failure effect. From a performance point of view, networks with centralized control can achieve better performance than with distributed control. However, with the increased size and density of Femtocell networks and the decentralized nature of Femtocells, it becomes difficult to use centralized algorithms.

In this work, we focus on the decentralized approach and run through several non-assisted and assisted techniques to cover different operational scenarios. Most known methods developed for downlink power control are either Non-assisted Techniques or Assisted Techniques. 


\subsubsection{Non-assisted techniques}

Fixed Femtocell power setting: a preconfigured value is common for all Femtocells regardless of the surrounding RF conditions. The advantages of this scheme are its simplicity and ease of implementation. Disadvantages are the difficulties to adapt to the surrounding RF conditions and the likeliness of causing large interference.

RSRP approach: the largest Reference Signal Received Power (RSRP) corresponding to the nearest Macrocell is used as one of the parameters for tuning downlink power control [30].

The previous techniques have not adequately accounted for the interference with neighbouring MUEs and the surrounding conditions.

\subsubsection{Assisted techniques}

Self-Optimization approach based on SINR: a self-optimization of coverage in accordance with the information on the mobility events of passing and indoor users is used [31].

Adaptation based on DL Reception Power from Macrocell: this technique is based on downlink co-channel reception power of the reference signal of the strongest Macrocell [32]. The Femtocell measures the reception power at the initial configuration phase or in the operational phase and adaptively sets the transmit power level accordingly:

$$
\text { P_tx }=\text { MEDIAN }(\text { Pm }+ \text { Poffset, P_tx_upp, P_tx_low })
$$

Where $\mathrm{Pm}[\mathrm{dBm}]$ is the reception power of the reference signal from the nearest Macrocell measured at the Femtocell and is dependent on the path loss between the nearest Macrocell and the Femtocell which includes the penetration loss at the building wall. Poffset $[\mathrm{dB}]$ is the predetermined fixed power offset compensating for the indoor loss. P_tx_upp and P_tx_low [dB] are the upper and the lower limit values of the transmit power. P_tx_upp is needed to limit the interference from the Femtocell to the Macrocell UE. P_tx_low is also needed to guarantee a certain minimum performance for the Femtocell even if the surrounding Macrocell cannot be detected. A disadvantage of this schema is that the fixed power offset is not enough to compensate for the indoor path loss. Each building has different properties, such as penetration loss at external walls and $\mathrm{P}$ offset should be tuned accordingly. 
Adaptation based on DL Reception Power from Macrocell and UL Reception Power from Macrocell UE: this technique is based on downlink (DL) cochannel reception power of the reference signal of the strongest Macrocell and uplink (UL) reception power from neighbouring MUEs [32]. The Femtocell adaptively measures the DL and UL reception power at self-configuration phase and then optimizes the transmit power during the self-optimization phase. The Femtocell sets the transmit power of the reference signal as follows:

$$
\mathrm{P}_{\mathrm{tx}}=\operatorname{MEDIAN}\left(\mathrm{P}_{\mathrm{m}}+\mathrm{P}_{\text {offseto }_{\mathrm{o}}}+\mathrm{K} * \mathrm{LE}, \mathrm{P}_{\mathrm{tx}_{\mathrm{upp}}}, \mathrm{P}_{\text {tx }_{\text {low }}}\right)
$$

Where $\mathrm{P}_{\mathrm{m}}, \mathrm{P}_{-} t \mathrm{x}_{\_}$upp and $\mathrm{P}_{-} t \mathrm{x} \_$low have the same meaning as in (2). $\mathrm{P}_{\text {offset }}$ $[\mathrm{dB}]$ is a predetermined power offset value compensating for the indoor path loss excluding the penetration loss. $\mathrm{K}$ is an adjustable positive factor and can be determined by the priority of the Femtocell operation. $\mathrm{LE}[\mathrm{dB}]$ is the penetration loss, which is assumed to be ideally estimated.

Auto-Tuning of DL Power of Femtocells Adaptive to Various Interference Conditions: the power offset in previously introduced techniques has not been adequately optimized for various interference conditions. The last proposed scheme automatically tunes the power offset so that the Femtocell throughput can increase while maintaining the Macrocell throughput based on Macrocell mobile stations' interference detection reports. Moreover, various interference conditions, such as the size of buildings where Femtocell mobile stations exist and distance to a street where Macro UEs exist are not sufficiently considered. Therefore, [33] introduces different auto-tuning schemes using individual offset where the transmit power is tuned individually per Femtocell, or using common offset where it is tuned commonly among Femtocells in a Macrocell with per-Femtocell or perMacrocell measurement, respectively. This approach uses a stepwise tuning of transmit power based on the Interference Detection Ratio (IDR1) indicator, which is the ratio of the number of interference detection reports to the number of Macro UEs that receive the measurement control message from the serving Macrocell.

A major disadvantage of the above techniques is that they do not properly consider the surrounding conditions and neighbouring Femtocells, especially in case of dense deployments (high number of Femtocell UEs and Femtocells located close to each other) and their intra-interference (across Femtocells). 


\section{Interference Management and Orchestration between Femtocells}

Once another Femtocell is set up nearby, the two signals of the two neighbouring Femtocells may interfere. There are some negotiation algorithms that are usually used to detect the interference, detect the interferer and run actual negotiation. Most of these algorithms follow one of following two approaches:

Centralized approach: assumes the existence of an O\&M unit that makes coordinated decisions of resource assignment for the Femtocells that report measurements to the unit. This unit can potentially orchestrate the behaviour of radio cells across an entire network of multi-technology environments. Since the control centralized, these interactions can be easily coordinated and managed using a variety of resolution techniques.

Decentralized approach: assumes that each Femtocell scans for other Femtocells on the available channels and estimates the pathlosses from those nodes. It is designed for near real-time response in seconds or milliseconds and therefore supports more frequent and localized changes than the centralized approach. Each node can initiate control processes and provide optimization decisions independently or in co-ordination with other nodes. This architecture makes the control functions highly dynamic and enables the network to adapt to local changes more rapidly.

The self-organization network (SON) techniques follow the same approaches for deploying small cells, as part of Heterogeneous Networks. Indeed, small cells must have plug-and-play simplicity, and they must be capable of automatically adapting their parameters (i.e., radio, mobility, security, transmit power, etc.) depending on conditions. Therefore, in LTE Release 10 and beyond, SON will continue to be a very important practice.

This can be either done in a centralized way, whereby a SON server optimizes a group of small cells, or it can be done in a distributed way, whereby each small cell optimizes its own parameters, as illustrated in Figure 4.

In practice, a combination of both is generally used, depending on the parameters, and is referred to as a hybrid cell. However, fully distributed SON will be the preferred approach in the future because it reduces complexity and costs. In our research work, we follow this approach and propose new algorithms based on distributed SON techniques.

To improve the signal strength for subscribed mobile devices without compromising signal quality, the Femtocell may use dynamic power levels, 


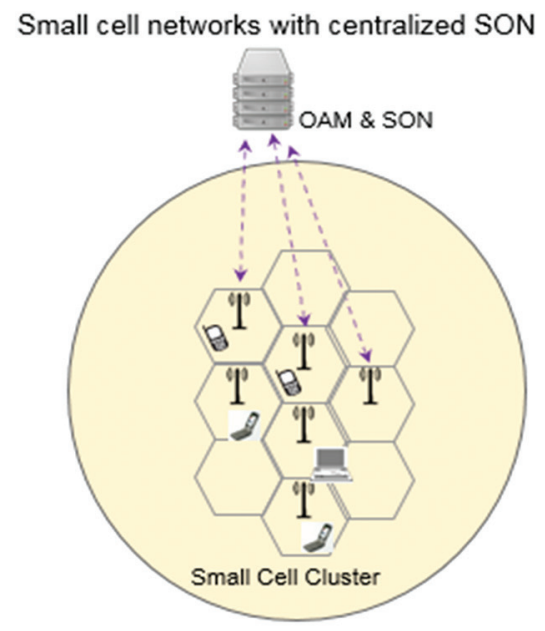

Figure 4 SON Centralized vs. Distributed Approaches.

depending on several factors like the distance between cells, path loss and penetration, subscribers' position, etc. In this work, we make use of these parameters and many more to achieve the best dynamic power settings of Femtocells in massive deployments.

\section{Novel Adaptive Power Sitting Frameworks}

In the scope of this work, we propose several downlink and uplink frameworks for adaptive power level settings to work efficiently in highly dynamic mobile environments. Comparing with existing solutions, we look to the end-to-end scenarios and their variant RF circumstances and changes. Our special focus is on dense deployments in HetNet and the best power control we can achieve which reduces the impact on environment. In comparison to the work done in the field, our first proposed framework for downlink takes into consideration intra-interference and dense Femtocell deployment situations, whereas the second considers additional measurement reports from the attached Femto UEs for further accuracy. The first proposed downlink framework is simpler and requires less signalling, whereas the second results in enhanced and more fine-tuned power settings. The light signalling overhead is analysed later in Chapter 6 (Evaluation Parameters). Both frameworks can be applied in different operational scenarios based on the Femtocell's ability to collect measurement reports from its UEs, hence the Femtocell should have a hybrid 
power setting mode and switch between the proposed frameworks according to the operation scenario. We also propose one adaptive power control framework for uplink where several types of uplink interference, affecting parameters, RF changing circumstances and dense/non-dense deployments are considered. It sets the transmit power by considering, for instance, the interference generated to neighbouring cells, the Overload Indication status of neighbouring cells and the incoming interference of neighbouring Femtocell and Macrocells and their users.

Our evaluation has shown the new frameworks to successfully preserve the users' service quality from Macrocells' and Femtocells' interference and converge to an optimal point under highly dynamic TX conditions.

\subsection{Downlink Distance-Based Framework}

This section introduces the first proposed DL adaptive power level setting framework in this paper. It takes into consideration intra-interference and dense Femtocell deployments. Femtocell should adjust the downlink transmit power by taking into account:

- Interference measurement from Macrocell.

- Interference measurement from neighbouring Femtocells.

- Path loss between the Femtocell and an outdoor neighbour MUE including penetration loss in order to provide better interference mitigation for the MUE while maintaining good Femtocell coverage for FUEs.

By measuring the signal strength of the sender, the receiving femtocell can estimate the path loss to the sender. Furthermore, using this path loss and the indicated power applied in each sub-channel, the receiver can estimate the received signal strength from the sender in each sub-channel. This is used for the calculation of the final compromising transmit power P_tx. The update procedure of transmit power value is performed periodically, and the time between consecutive updates is randomly chosen from the interval of $[1,2 \mathrm{~T}]$ time units. This is done in order to avoid that several Femtocells change their power settings at the same time. Between updates, the Femtocell senses the power strengths from its neighbours. These are processed at the next update, in which the Femtocell first re-computes the power level based on existing factors. Afterwards, the Femtocell rearranges its power level so that the most optimal values are used.

Figure 5 shows the interference factors between Femtocell and Macrocell, Femtocell and Macrocell MS and neighbouring Femtocells. The Femtocell 
should set the transmit power of reference signal P_tx as follows:

$$
\mathrm{P}_{\mathrm{tx}}(\mathrm{dBm})=\operatorname{MEDIAN}\left(\mathrm{P}_{\mathrm{m}}+\mathrm{P}_{\mathrm{m}_{\text {offset }}}, \mathrm{P}_{-} \mathrm{f}+\mathrm{P}_{\mathrm{f}_{\text {offset }}}, \mathrm{P}_{\mathrm{tx}_{\mathrm{upp}}}, \mathrm{P}_{\mathrm{tx}_{\text {low }}}\right)
$$

Where P_m $(\mathrm{dBm})$ is RSRP from the nearest Macrocell measured by the Femtocell, P_m is dependent on path loss which includes the penetration loss between the nearest Macrocell and the Femtocell. P_m_offset (dB) is the power offset. It is defined based on path loss between the Femtocell and the MUE. The path loss may consist of indoor path loss between the Femtocell and its cell edge and the penetration loss. Therefore P_m_offset should be formulated as $\left(\mathrm{P}_{\text {offset }_{\circ}}+\mathrm{K} * \mathrm{LE}\right)$ determined in (3) before. $\mathrm{P}_{-} \mathrm{f}$ $(\mathrm{dBm})$ is the highest RSRP from the neighbouring Femtocells measured by the Femtocell. P_f is dependent on path loss which includes the penetration loss to the neighbouring Femtocells. P_f_offset $(\mathrm{dB})$ is the predetermined fixed power offset compensating for the indoor loss. P_tx_upp/P_tx_low $(\mathrm{dBm})$ is the upper/lower limit value for the transmit power of the reference signal. This framework outperforms by taking into account interference measurement
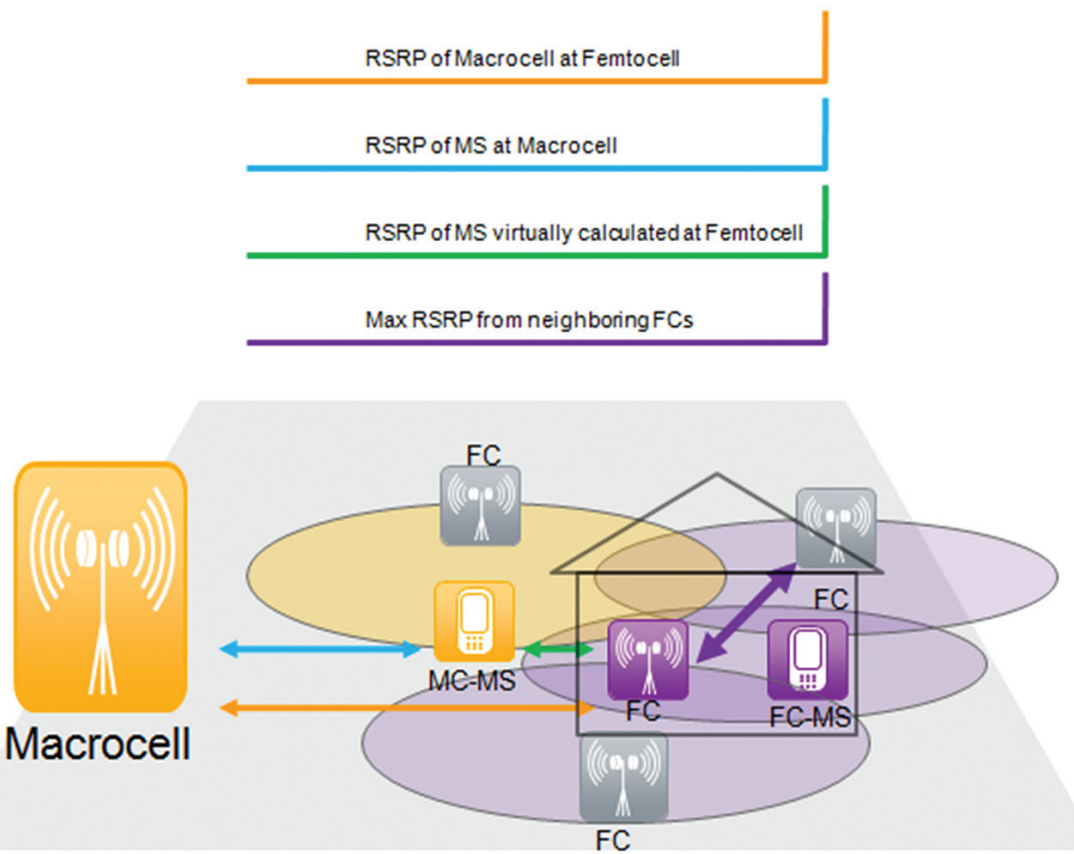

Figure 5 Penetration Loss between Femto-Macro, Macro-MUE and neighbouring Femtocells. 
from neighbouring Femtocells. This is very crucial in case of dense deployment where hundreds or thousands of Femtocells are located close to each others.

When a power update request is due in $\mathrm{Fi}$, it gathers the information of all neighbouring Femtocells and builds a RSRP matrix M. The dimensions of this matrix $\mathrm{M}$ are $\mathrm{Di} \times \mathrm{G}$, where Di refers to the number of sensed neighbouring Femtocells by $\mathrm{Fi}$ and $\mathrm{G}$ is the number of sub-channels. Once that the RSRP matrix $\mathrm{M}$ is built, Femtocell Fi re-computes its transmit power considering the following procedure, whose target is to find out the maximum (highest) RSRP of the probably closest neighbouring Femtocell.

$$
\begin{array}{r}
P_{-} f=\operatorname{Max} \sum_{x=0}^{D i-1} \sum_{i=0}^{G-1} M_{d, g} \cdot \beta_{d, g} \\
\beta_{d, g} \in\{0,1\} \quad \forall_{d, g}
\end{array}
$$

Where $\beta_{\mathrm{d}, \mathrm{g}}$ is a binary variable that is equal to 1 if user $\mathrm{d}$ is using sub-channel $\mathrm{g}$, and 0 otherwise.

\section{Proposed Algorithm}

INITIALIZE power control request every $[1,2 \mathrm{~T}]$ time unit

WHILE (neighboring Femtocells)

Sense the neighboring Femtocells \& their UEs

IF (number of neighboring Femtocells $>0$ ),

Calculate the RSRP matrix

Calculate the highest RSRP P_f of the neighboring Femtocells

WHILE (neighboring Macrocells)

Sense the neighboring Macrocells \& their UEs

IF (number of neighboring Macrocells $>0$ )

Calculate RSRP from the nearest Macrocell

IF (Macro UE)

Macro UE

Calculate PL between Femtocell and outdoor neighboring

Calculate P_m_offset (according to Poffseto, K, LE)

SET P_tx value and the transmit power to use

\subsubsection{Neighbouring Femtocells}

Femtocell $\mathrm{Fi}$ is considered neighbour of Femtocell $\mathrm{Fj}$ if they are in the range of each other (there is 'visibility'), i.e., the received signal strength $R_{i, j}$ 
coming from Femtocell Fi is larger than the sensitivity $\emptyset_{j}$ of the antenna of Femtocell Fj:

$$
\emptyset_{j}<R_{i, j}=P_{i, k} * G_{i} * L_{i} * P L_{i, j} * G_{j} * L_{j}
$$

Where $\mathrm{k}$ is the sub-channel; $\mathrm{i}$ is the index of the transmitting Femtocell Fi; $\mathrm{j}$ is the index of the receiving femtocell, $\mathrm{Fj} ; \mathrm{Pi}, \mathrm{k}$ is the power applied by $\mathrm{Fi}$ in a sub-carrier of sub-channel $\mathrm{k} ; \mathrm{PLi}, \mathrm{j}$ represents the path loss attenuation and shadow fading between $\mathrm{Fi}$ and $\mathrm{Fj} ; \mathrm{G}$ stands for the antenna gains and $\mathrm{L}$ for the equipment losses.

\subsection{Downlink Measurement Reports-Based Framework}

This section introduces our second proposed framework in this paper which is additionally consider UE measurement reports. The measurement of Macro and neighbouring Femtocells received power alone is not sufficient in some scenarios when Femtocell and FUEs are located in different rooms, floors, etc. with different penetration and path loss conditions. In some cases, the Femtocell may be using a higher power level than required (see Figure 6). Therefore, we need to consider measurement reports from the Macrocell, MUE, neighbouring Femtocells and connected FUEs as well. This will enable us to accurately determine the values for the Femtocell power settings. The Femtocell should dynamically adjust the downlink transmit power by taking into account: (a) path loss between the Femtocell and the outdoor neighbour MUE, including penetration loss, in order to provide better interference mitigation for the MUE while maintaining good Femtocell coverage for FUEs. (b) Path loss to the attached indoor FUEs and their possible measurement reports. (c) Interference measurement from the Macrocell and neighbouring Femtocells. This framework doesn't entail modifications on the end host OS.

The Femtocell should set the transmit power of reference signal $P_{-}$tx as follows:

$$
\begin{aligned}
& \text { P_tx }(\mathrm{dBm})=\max \left(\mathrm { P } _ { \_ } \mathrm { tx } \_ \text { low, } \operatorname { m i n } \left(\operatorname { M E D I A N } \left(\mathrm{P} \_\mathrm{m}+\mathrm{P} \_\mathrm{m} \_\right.\right.\right. \text {offset, }
\end{aligned}
$$

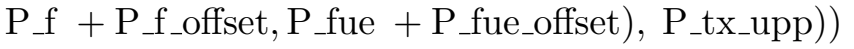

Where $\mathrm{P} \_\mathrm{m}(\mathrm{dBm})$, P_offset_m $(\mathrm{dB}), \mathrm{P} \_\mathrm{f}(\mathrm{dBm}), \mathrm{P} \_$_offset $(\mathrm{dB}), \mathrm{P}_{-} \mathrm{tx} \_$upp and $P_{-}$tx_low $(\mathrm{dBm})$ are explained in $(4)$. $P_{-}$fue $(\mathrm{dBm})$ is the minimum reported RSRP from the Femto UEs. P fue is dependent on path loss that includes the penetration loss between the Femtocell and its FUEs. P_fue_offset (dBm) 


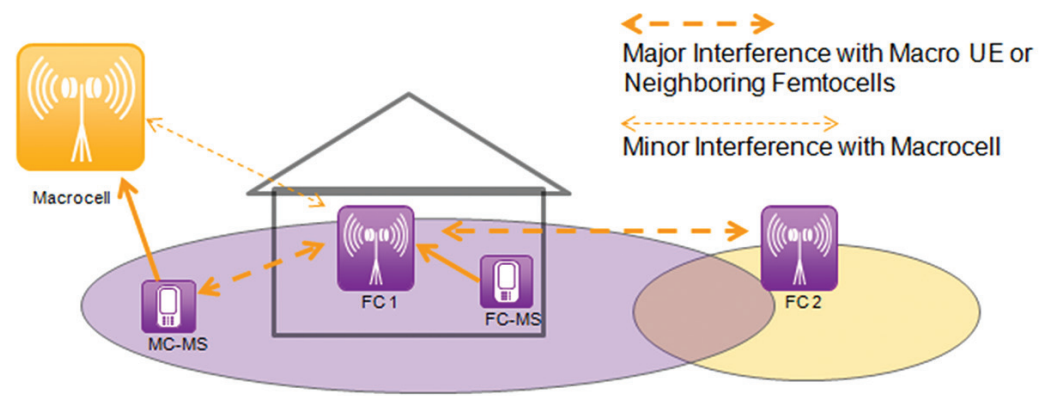

(a)

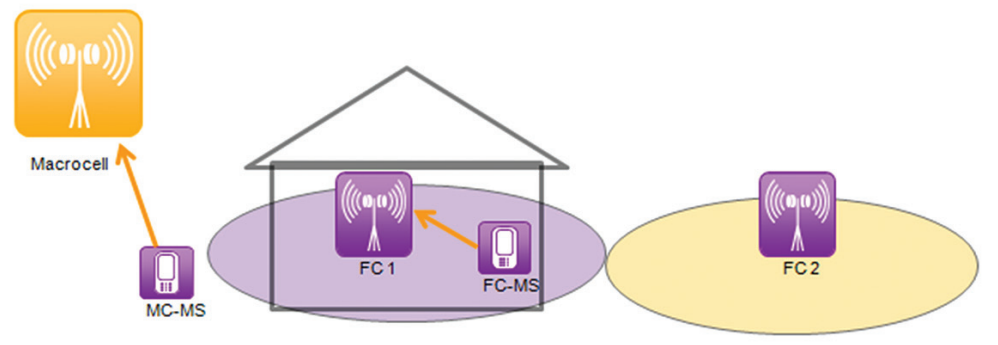

(b)

Figure 6 Scenarios where Femtocell transmit power needs to properly set. (a) Scenarios where Femto Tx Power is not properly set taking into consideration HUE received signal, (b) Femto Tx Power is properly set taking into consideration HUE received signal.

denotes a power offset value that captures the indoor Path loss and penetration loss between the Femtocell and its FUEs. Since FUE is attached to the Femtocell, FUEs can periodically report the measurement information to its serving Femtocell, which will result in enhanced and accurate power setting.

In this approach, a user UE sends a Measurement Report (MR) to its serving Femtocell Fi every $\mathrm{T}$ time units. MR indicates the received signal strength suffered by user UE. Then, Femtocell Fi updates its transmit power according to the received MRs. The adjustment of operational power setting happens after a random time interval between 1 and $2 \mathrm{~T}$ time units after the last update event. In this way, several Femtocells avoid changing their transmit power at the same time, enhancing the coordination.

When a power update needs to happen in $\mathrm{Fi}$, it gathers the information of all received MRs and calculates all the values required for Equation (6) like P_m, P_f, P_fue, etc. For the calculation of P_fue, it builds a RSRP matrix S. The dimensions of this matrix $\mathrm{S}$ are $\mathrm{Ni} \times \mathrm{K}$, where $\mathrm{Ni}$ refers to the number 
of neighbouring users to Femtocell Fi and $\mathrm{K}$ is the number of sub-channels. Once that the RSRP matrix $\mathrm{S}$ is built, Femtocell Fi re-computes its transmit power considering the following procedure, whose target is to find out the lowest required RSRP of the highest path loss suffered by Femtocell users.

$$
\begin{array}{r}
\mathrm{P} \_ \text {fue }=\operatorname{Min} \sum_{\mathrm{x}=0}^{\mathrm{Ni}-1} \sum_{\mathrm{i}=0}^{\mathrm{K}-1} \mathrm{~S}_{\mathrm{n}, \mathrm{k}} \cdot \beta_{\mathrm{n}, \mathrm{k}} \\
\beta_{\mathrm{n}, \mathrm{k}} \in\{0,1\} \quad \forall_{n, k}
\end{array}
$$

Where $\beta_{\mathrm{n}, \mathrm{k}}$ is a binary variable that is equal to 1 if user $\mathrm{n}$ is using sub-channel $\mathrm{k}$, and 0 otherwise. The power setting framework proposed in (6) provides more accurate interference mitigation for neighbouring MUE, Macrocell and other Femtocells and maintains good Femtocell coverage for FUEs on an accurate power level, especially in Femtocell-dense deployments and without affecting the FUE throughput negatively.

\section{Proposed Algorithm}

INITIALIZE power control request every $[1,2 \mathrm{~T}]$ time unit

WHILE (neighboring Femtocells)

Sense the neighboring Femtocells \& their UEs

IF (number of neighboring Femtocells $>0$ ),

Calculate the RSRP matrix

Calculate the highest RSRP P_f of the neighboring Femtocells

WHILE (neighboring Macrocells)

Sense the neighboring Macrocells \& their UEs

IF (number of neighboring Macrocells $>0$ )

Calculate RSRP from the nearest Macrocell

IF (neighboring Macro UE $>0$ )

Macro UE

Calculate PL between Femtocell and outdoor neighboring

Calculate P_m_offset (according to Poffseto, K, LE)

IF (number of Femto UEs $>0$ )

Calculate the minimum required RSRP of the highest path loss

Calculate P_f_offset

Calculate P_fue_offset

SET P_tx value and the transmit power to use 


\subsection{Uplink Enriched FPC Based Framework}

Under the expected co-channel Femtocell deployment, UL transmissions of Femtocell users may cause interference to the ongoing UL transmissions of users being served by Macrocells or nearby Femtocells of other Macro clusters, and vice versa [21, 22]. The open loop power control formula for UL is standardized, while the setting of its parameters (i.e., $P_{0}$ and $\alpha$ ) is open and up to network operators. Our intent is to proactively improve the SINR (Quality) for the scheduled users by automatically adjusting those power control parameters. This will improve the resource scheduling experience and reduce the interference and power consumption at both Cell and UE levels.

From the Macro point of view, in order to avoid blinding the Femtocell completely due to Macrocell user uplink power, power control algorithms have been introduced for Macrocell users. They take into consideration the distance of the Macrocell user from Macrocell base station and allocate appropriate uplink power to that user. However, we believe this is not enough and, in the case of Femtocell co-deployment, the following factors must be considered too:

- For FUE, Interference/Distance to Macrocell base station and serving Femtocell.

- Interference/Distance to other surrounding Femto UEs (not belonging to the same Macro cluster).

- Interference/Distance to other surrounding Macro UEs (for the sake of simplicity, we assume receiving this measure from the serving Femtocell).

The FPC (Fractional Power Control) scheme allows a user to be received with variable PSD depending on their path loss; i.e., a user with good radio conditions will be received with high PSD. In contrast to conventional power control, where $\alpha=1$, or no compensation at all with $\alpha=0$, FPC compensates for the fraction of the path loss.

In most existing power setting approaches, both $\mathrm{P}_{0}$ and $\alpha$ values are fixed broadcasted cell specific towards the UE, meaning that they are same for all the UEs (thus PL is the only key factor).

We propose a dynamic calculation of $\mathrm{P}_{0}$ and $\alpha$ that allows users to be received with different power spectral densities based on their own RF quality characteristics. Our approach is to set the UL transmit power by considering different factors like the interference generated to neighbouring cells and UEs, as well as the Noise Interference (NI) status of adjacent cells (Traffic Situation), so that we adaptively adjust the transmit power in order to avoid full collapse 
effects or give the passing MUE some privilege above the Femtocell that is not really loaded.

In this work, we analyse the influence of the compensation factor in related work presented in Equation (1); ignoring Pmax, $\triangle \mathrm{TF}$ and $\mathrm{f}$, we consider a single RB $(M=1)$. In this simplified case, transmit power PTx can be written (in $\mathrm{dBm}$ ):

$$
\operatorname{PSDTx}=\mathrm{P} 0+\alpha \cdot \mathrm{PL}[\mathrm{dBm} / \mathrm{PRB}]
$$

And the received power PRx at the hosting cell:

$$
\operatorname{PSDTx}=\mathrm{P} 0+\alpha \mathrm{PL}-\mathrm{PL}=\mathrm{P} 0+(\alpha-1) \mathrm{PL}
$$

$\mathrm{P}_{0}$ and $\alpha$ are used to control the average received Power Spectral Density (PSD) at Cell level, and thereby the target SINR. $\mathrm{P}_{0}$ is the compensation factor of target received power at cell level and can be defined as follows ( $\mathrm{dBm}$ scale):

$$
\mathrm{P}_{0}=\alpha *(\mathrm{SNRi}+\mathrm{IN})+(1-\alpha) *(\mathrm{Pmax})[\mathrm{dBm} / \mathrm{PRB}]
$$

If UE is identified as Cell Edge UE, then low SNRi should be used; otherwise it should be high. Hence, for a 'cell-edge' UE, using $\mathrm{M}=\mathrm{M} 0 \mathrm{RBs}$, to reach a desired SINR, the associated P0 value can be found by:

$$
\mathrm{P}_{0}=\alpha(\mathrm{SNRi}+\mathrm{NI})+(1-\alpha) *(\mathrm{Pmax}-10 \log \mathrm{M} 0)
$$

Where SNRi is a fixed positive integer based on interference (UE position) and the neighbouring Traffic situation (important in case of Cell Edge UEs), NI is a compensation value for the noise figure and the rise of interference over the thermal noise. If the neighbouring Femtocell can accept high interference, then SNRi for the UE can be set higher, and vice versa. Cell coordination with interference and load information sharing help more suitable SINR target setting. The status of the neighbouring cell is used to adjust the SINR target, which has two advantages, 1) improve performance when neighbouring cell has low overload level; 2) avoids full collapse when the neighbouring cell has a high overload level.

We see that increasing $\alpha$ will increase the received power of not only the cell edge UE but also the inter-cell interference. So $\alpha$ should be set in order to maximize cell capacity, minimize inter-cell interference, save power and keep an acceptable level of SINR.

The solution proposed in this study is to configure the OLPC parameter $\alpha$ for the UE in a way that would dynamically reflect the distribution of interference levels within the coverage $\&$ surrounding areas (adaptive $\alpha$ ) as 
a function of received power from closest Macro-eNB, surrounding other Femtocells and neighbouring Femto \& Macro UE. The more interference measured from the surrounding environment the less $\alpha$ should be and less PL is included in the power calculation. This will enable us to consider edge UE situation and neighbouring cells. In the case of a light-loaded neighbouring cell, $\alpha$ will go higher to prioritize Macro UE. When the measured interference goes to 0 then $\alpha$ goes to 1 and when the interference goes to 8 then $\alpha$ goes to 0 :

$$
\alpha=\operatorname{abs}(\text { interference/(interference }+\mathrm{NI})) ; \alpha \epsilon[0-1]
$$

Where interference $\epsilon[0-\infty]$ and calculated based on max measured RSRP received from Macrocell, neighbouring Femtocell, MUE and neighbouring FUE of a different Macro cluster. NI is the Noise Interference indicator of interfering neighbouring cells.

In our approach, the identification of Cell Interior and Edge UEs will take into account the path loss to both serving cell and neighbouring cell. For the identification of cell edge UE, we assume that for each UE the serving cell and closest neighbouring cell can be identified via DL received pilot power. Path loss difference $\triangle P L=P L \_$neighbour - $P L \_$serving is more reasonable than $P L \_$serving alone to identify cell edge from cell interior UEs.

Cell interior UE with large path loss difference contributes less interference to neighbouring cells, thus a high SINR target can be set for them; while, for cell edge UE with small path loss difference like MUE-2 in Figure 7, high

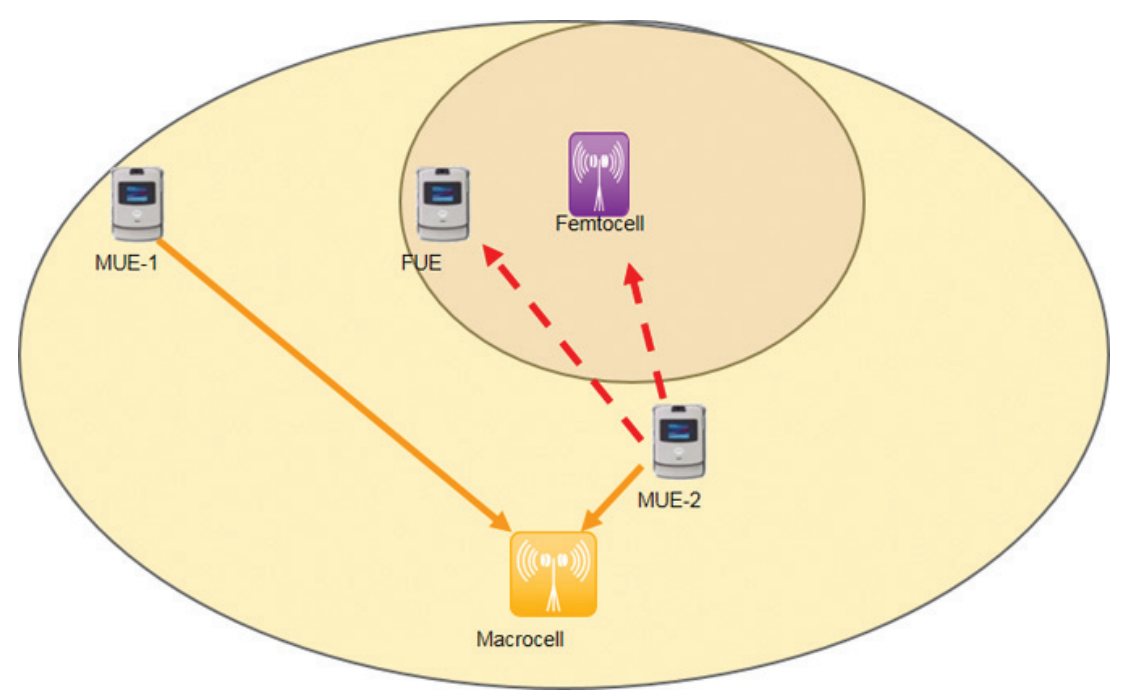

Figure 7 Identification of cell edge UE and cell interior UE. 
interference is generated to neighbouring cells and a low SINR target should be set for them.

\section{Proposed Algorithm}

\section{DETECT power control request}

WHILE (interfering neighbouring cells)

Sense the neighbouring Macrocells, Femtocells \& their Ues

\section{CASE of MUE}

Calculate signal strength between user and 1) serving cell 2) neighboring Femtocells

\section{CASE of FUE}

Calculate signal strength between user and 1) serving cell 2) neighboring Macrocells 3) Femtocells that don't belong to the same Macrocell

IF (number of neighbouring Cells $>0$ ),

SELECT neighbor cell with the highest RSRP (probably closest) Calculate deltePL $=$ PL_neighbor (CELL/UE) - PL_serving IF delta PL $>$ PLth* (UE is cell edge UE)

Collect the load (OI) and IoT of Cell_i as L_i

Calculate alpha \& P0 according to Load situation of neighboring cells (alpha is fractional based on load \& between 0 and 1)

ELSE (UE is cell interior UE)

\section{ELSE}

Calculate alpha \& P0 according to received power

Calculate alpha \& P0 according to received power SET P_tx value and the transmit power to use

In the algorithm L_i denotes the overload of the corresponding Cell. We also considered a threshold PLth which is used to identify the UE type (Cell edge or interior UE).

To keep the additional required signalling low and avoid more processing at the UE side, which means higher power consumption and less battery life, we run our proposed UL enhanced FPC at serving cell which will adjust the transmission power of individual UEs.

\section{Evaluation Parameters}

LTE-Sim system-level simulator in [35] is used in our study cases. $5 \times 5$ apartment grid building types, shown in Figure 8 below, are chosen where 


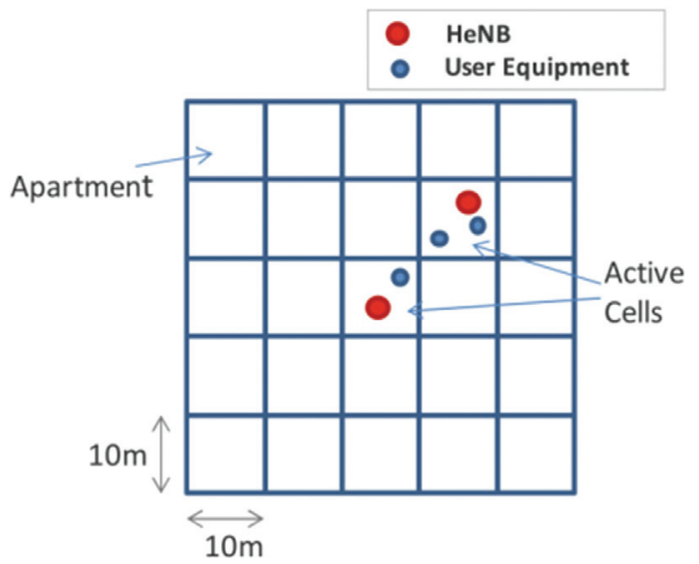

Figure $85 \times 5$ apartment grid building type.

each building is composed of 25 apartments located over a $5 \times 5$ grid. Each apartment has a squared form and an area of $100^{2}$.

Different number of Macro- and Femtocells and their users are chosen according to required density of the simulated scenario. Random mobility model is used with UE speed for about $3 \mathrm{~km} / \mathrm{h}$.

\subsection{Throughput Calculation}

Throughput is computed of a given flow as the ratio between the sum of bits correctly received and the simulation time.

\subsection{Propagation Loss}

The Channel Module of the simulator handles packet transmissions and models the propagation loss by means of four different phenomena as suggested in [36]: (i) the path loss, (ii) the penetration loss, (iii) the shadowing, and (iv) the fast fading due to the signal multipath.

For a user located indoors and served by a Macrocell, the path loss calculation also takes into consideration an additional attenuation factor due to the presence of an external wall (default value of the external wall attenuation is $20 \mathrm{~dB}$ [37]).

$$
\begin{array}{r}
\mathrm{PL}[\mathrm{dB}]=128.1+37.6 * \log 10(\text { Distance } * 0.001) \\
+ \text { external wall attenuation }
\end{array}
$$


Moreover, in order to cope with the peculiar features of Femtocells' dense deployments, the path loss model in (12) is also used to evaluate the path loss between neighbouring Femtocells.

The Path Loss [dB] from Femtocell to UE developed within the WinnerII project [38] for indoor residential environments is used. It offers a high accuracy at the cost of an increased computational complexity:

$$
\mathrm{PL}[\mathrm{dB}]=\mathrm{A} * \log 10(\mathrm{R})+\mathrm{B}+\mathrm{C} * \log 10(\mathrm{fc} / 5)+\mathrm{X}
$$

Where $\mathrm{R}$ is the distance expressed in meters; the central frequency fc is expressed in gigahertz; the values of other parameters A, B, and C depend on the number of walls and floors between the transmitter and the receiver. $\mathrm{X}$ is the sum of the internal and external walls' attenuation.

\subsection{Green Impact Calculation}

In [39], a new indicator is introduced that enables us to calculate the $\mathrm{CO}_{2}$ emissions consumed per $\mathrm{kWh}$ and highlights the green effect of the saved energy. Based on guidelines on conversion factors for company reporting, produced by the Department of Energy and Climate Change and the Department for Environment, Food and Rural Affairs (DECC \& DEFRA), the kWh is converted to $\mathrm{kg}$ of carbon saved. For instance, the conversion factor for Italy is $0.46225 \mathrm{~kg} \mathrm{CO}_{2}$ saved for each $\mathrm{kWh}$ produced from a carbon-free source. In other words, each $\mathrm{kWh}$ of electricity coming from the grid in Italy produces roughly $462.25 \mathrm{~g}$ of $\mathrm{CO}_{2}$. The factor is based on the carbon emissions generated by Italian power stations per $\mathrm{kWh}$ generated. This factor includes other greenhouse gasses such as methane and nitrous oxide, which are converted to their carbon dioxide equivalents so the value is really $\mathrm{kg}$ $\mathrm{CO}_{2}$ eq. per $\mathrm{kWh}$. The $\mathrm{CO}_{2}$ consumption can be calculated as shown in the following equation:

$$
\mathrm{CO}_{2}(\mathrm{~kg} .)=\text { Power }(\mathrm{kWh}) \times \text { Conversion factor }
$$

Alternatively, the energy demands of different base station types are introduced in [40]. This includes the $\mathrm{CO}_{2}$ emissions consumed per Watt and the yearly operative expenses (OPEX) due to power consumption. According to the latest estimates from the International Energy Agency and using Italy as a reference point, each $\mathrm{kWh}$ of electricity coming from the grid produces roughly $386 \mathrm{~g}$ of $\mathrm{CO}_{2}$ emissions. The real $\mathrm{CO}_{2}$ saving in Renewable Energy Systems (RES) 
powered HetNet is estimated by subtracting the amount of carbon emissions generated when realizing a PV (photovoltaic) system from the $\mathrm{CO}_{2}$ emissions due to the grid.

In [41] the power need (watt) of a Femtocell is estimated to be 10.4 watt in full load mode and 9.6 watt in idle mode. We have used the indicators introduced in [39] and [40] to explain the green effect of our new Femtocell comprehensive power control frameworks.

\subsection{Overhead (Signalling) Analysis}

In the case of using the Downlink Distance-Based method, the required uplink (UL) and downlink (DL) bandwidth for the distance sensing overhead is 0 , whereas in the case of using the Downlink Measurement Reports-Based method, the required UL bandwidth for the measurement reports overhead is equal to $\mathrm{M}=\mathrm{k} * \mathrm{~b} * \mathrm{u} * \mathrm{fr}$, whereas the required DL bandwidth is 0 . In this case, $b$ indicates the number of bits required to encode the received signal strength measured in a given sub-channel, $u$ denotes the number of connected users to the Femtocell, and $k$ indicates the number of sub channels. Finally, $f r$ is the reporting frequency.

Assuming a configuration where the updating and reporting frequency is $1 \mathrm{~s}-1$, there are 8 sub-channels, 4 connected UEs and that every transmitted value is encoded using 8 bits, the UL (DL) bandwidth overhead requirement of the measurement reports is $0.256 \mathrm{kbps}(0.00 \mathrm{kbps})$ respectively.

Therefore, it can be concluded that only a small fraction of the whole available bandwidth is needed for signalling overhead M. Note that, contrary to the Femtocell case, in Macrocell scenarios the processing of the measurement reports constitutes a significant overhead, since hundreds of users can be simultaneously connected to the Macrocell. Moreover, the channel conditions of Macrocell users change faster due to their higher mobility and the large number of obstacles existing in the environment. Measurement reports must be sent more often in order to cope with these fluctuations, but this increases the overhead. In this regard, the signalling overhead in case of Femtocell is very light.

\subsection{Key Parameters}

Key simulation parameters for our Femtocell DL power control frameworks are listed in Tables 1 and 2. 
Table 1 Macrocell key simulation parameters

\begin{tabular}{ll}
\hline Parameter & Macro Parameters \\
\hline Macrocell Radius & $1 \mathrm{~km}$ \\
Carrier Bandwidth & $20 \mathrm{MHz}$ \\
Path loss [dB] from Macro to MUE & $128.1+37.6 * \log 10($ distance $* 0.001)$ and if \\
& MUE is indoor, we add external wall attenuation \\
Path loss [dB] from Macro to & $128.1+37.6 * \log 10($ distance $* 0.001)+$ external \\
Femtocell/FUE & wall attenuation \\
Nr. of MUE & $1 / 25$ \\
Nr. of Macro cells & 1 \\
Total Macrocell TX Power & $43 \mathrm{dBm}$, equally distributed among sub-channels \\
Min. distance between Macrocell and & $>=35 \mathrm{~m}$ \\
Femtocell & \\
MUE speed & $3 \mathrm{~km} / \mathrm{h}$ \\
Distribution of MUS within Macrocell & Uniform \\
\hline
\end{tabular}

Table 2 Femtocell key simulation parameters

\begin{tabular}{ll}
\hline Parameter & Macro and Femto Parameters \\
\hline Femtocell Frequency Channel and & Same frequency and bandwidth as Macrocell \\
Bandwidth & layer \\
Path loss [dB] from Femtocell to & $128.1+37.6 * \log 10($ distance $* 0.001)+$ external \\
neighbouring Femtocell & wall attenuation \\
Path Loss [dB] from Femtocell to UE & A $* \log 10($ distance $)+\mathrm{B}+\mathrm{C} * \log 10(\mathrm{fc} / 5)+\mathrm{X} ; \mathrm{fc}$ \\
& is the central frequency expressed in gigahertz; A, \\
& B, and C values depend on the number of walls \\
& and floors between the transmitter and the \\
& receiver. X is the sum of Internal and External \\
& Walls Attenuation \\
P_offset_o & Between 50 and $110 \mathrm{~dB}$ \\
P_f_offset & Between 100 and 135 dB \\
K & 1 \\
La & $100 \mathrm{~dB}$ \\
Distribution of FUE within Femtocell & Uniform \\
Min distance between FUE and Femtocell & $>=10 \mathrm{~cm}$ \\
Parameter & Macro and Femto Parameters \\
Apartment size & $100 \mathrm{~m}{ }^{2}$ \\
Nr. of apartment on a building & 25 \\
Nr. of buildings & 1 (non-dense) or 2 (dense) \\
Building Type & $5 \times 5$ grid \\
Nr. of Femtocells & 1 per apartment (25 per Building) \\
Total Femtocell TX Power & $20 \mathrm{dBm}$, equally distributed among sub-channels \\
Nr. of FUE & $25 / 50$ \\
Speed of UE & $3 \mathrm{~km} / \mathrm{h}$ \\
\hline
\end{tabular}


Table 2 Continued

\begin{tabular}{ll}
\hline Parameter & Macro and Femto Parameters \\
\hline Flow Duration & 20 Second \\
Frequency reuse schema & Reuse-1 \\
Activity Factor & 1 \\
Scheduling Type & Proportional Fair (PF) \\
Traffic & (Best Effort Flows) Infinite Buffer \\
Access Policy & Open Access \\
Frame Structure & Frequency Division Duplex (FDD) \\
External Walls Attenuation & $20 \mathrm{~dB}$ \\
Internal Walls Attenuation & $10 \mathrm{~dB}$ \\
\hline
\end{tabular}

\section{Performance Evaluation}

This section presents performance comparison between the proposed frameworks in this work and other earlier power control models. This performance evaluation has been carried out using the system-level simulator explained in Chapter 6.

Regarding the downlink proposed frameworks, two other power control models have been considered to compare with: Fixed power control model and Adaptive one based on interference measurement from the nearest Macrocelland MUE path loss. The simulated UEs (Femto and Macro related) are moving randomly with a speed of $3 \mathrm{~km} / \mathrm{h}$. We mainly analyzed the impact on UE throughput (Mbps) and Femtocell power consumption $(\mathrm{dBm})$ in hybrid-dense deployments.

\subsection{Downlink Distance-Based Framework}

Two different usecases are object of the study for non-dense deployment:

- Usecase 1: 1 Macrocell and 25 Femtocells setup with moving 1 Macrocell UE and 25 Femtocell UEs considered - see Figures 9 and 10 (left-side).

- Usecase 2: 1 Macrocell and 25 Femtocells setup with moving 25 Macrocell UE and 50 Femtocell UEs considered - see Figures 9 and 10 (right-side).

Two usecases are object of the study for dense deployment (more Femtocells/area):

- Usecase3: 1 Macrocell and 50 Femtocell in dense deployment with moving 1 Macrocell UE and 25 Femtocell UEs considered - see Figures 11 and 12 (left-side). 


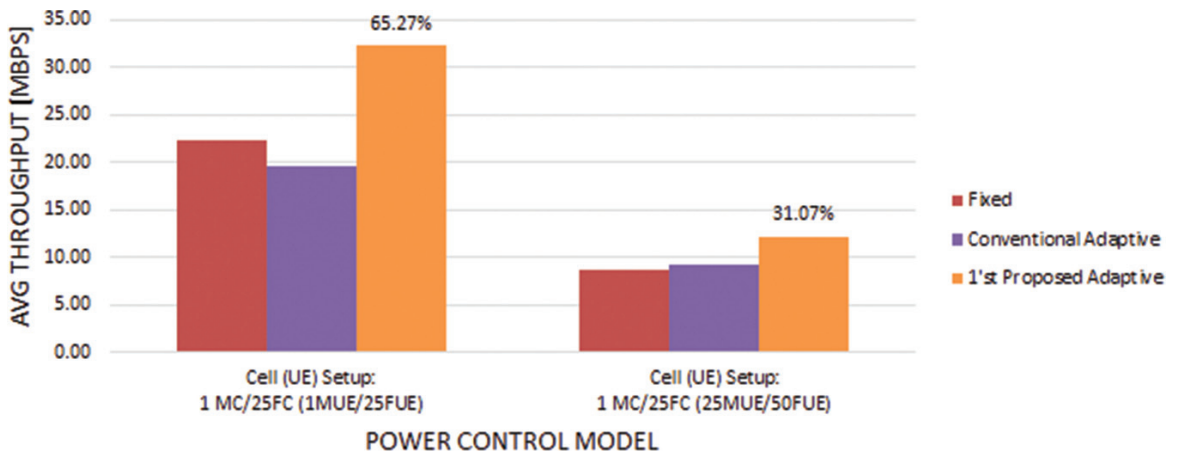

Figure 9 Average UE throughput in urban scenario and non-dense deployment.

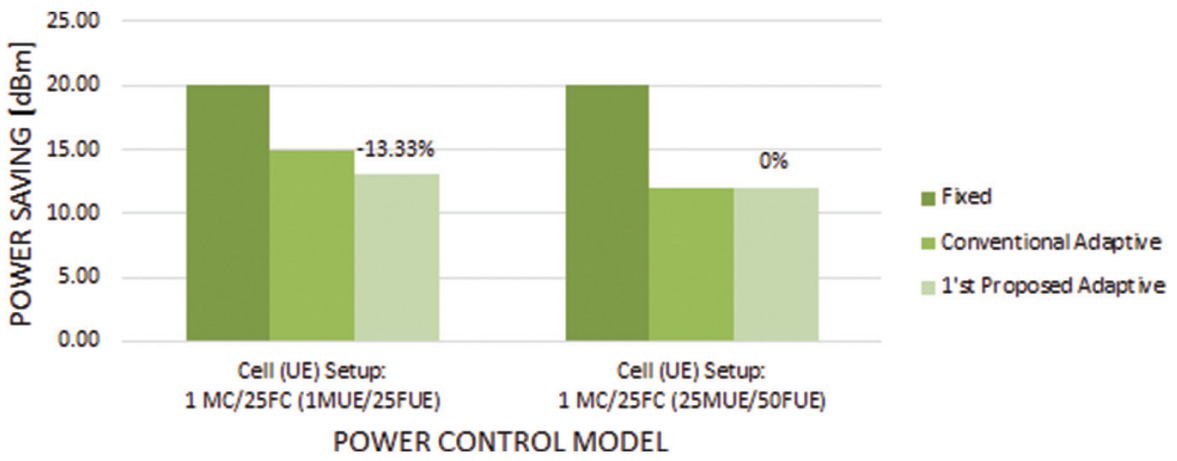

Figure 10 Power consumption per Femtocell in urban scenario and non-dense deployment.

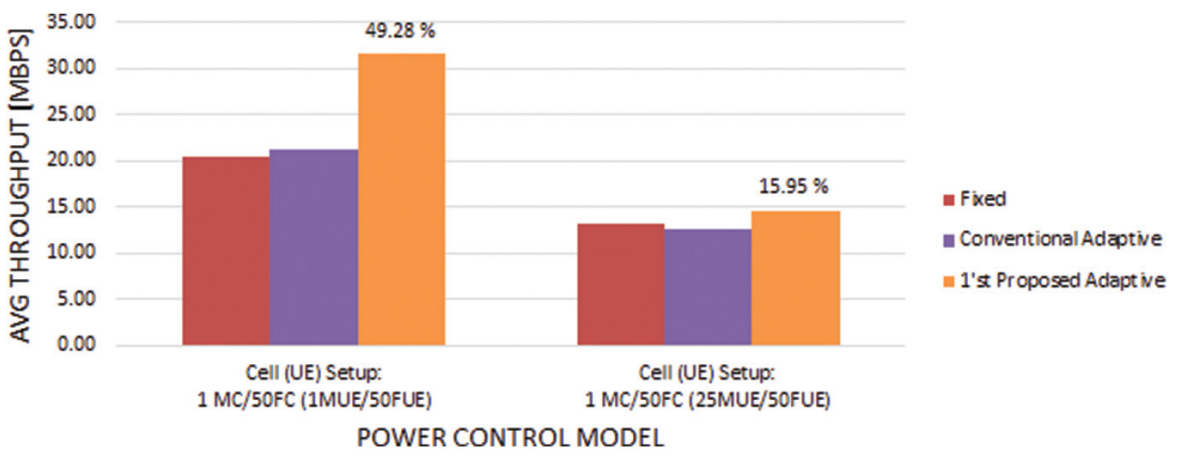

Figure 11 Average UE throughput in urban scenario and dense deployment. 


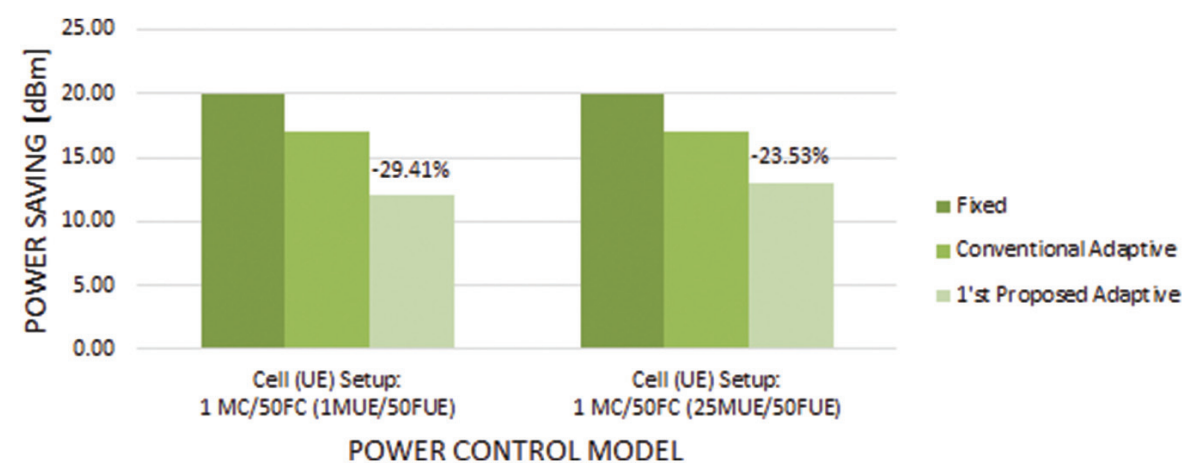

Figure 12 Power consumption per Femtocell in urban scenario and dense deployment.

- Usecase 4: 1 Macrocell and 50 Femtocell in dense deployment with moving 25 Macrocell UE and 50 Femtocell UEs considered - see Figures 11 and 12 (left-side).

As a result we can see that the throughput has improved and better power consumption and energy efficiency have been achieved after introducing our new power control paradigm in Femtocell in dense as well in non-dense deployments.

\subsection{Downlink Measurement Reports-Based Framework}

Two usecases are object of the study for non-dense deployment:

- Usecase 5: 1 Macrocell and 25 Femtocells setup with moving 1 Macrocell UE and 25 Femtocell UEs considered - see Figures 13 and 14 (leftside).

- Usecase 6: 1 Macrocell and 25 Femtocells setup with moving 25 Macrocell UE and 50 Femtocell UEs considered - see Figures 13 and 14 (right-side).

Two usecases are object of the study for dense deployment (more Femtocells/area):

- Usecase 7: 1 Macrocell and 50 Femtocell in dense deployment with moving 1 Macrocell UE and 50 Femtocell UEs considered - see Figures 15 and 16 (left-side).

- Usecase 8: 1 Macrocell and 50 Femtocell in dense deployment (several buildings) with 25 Macrocell UE and 50 Femtocell UEs considered - see Figures 15 and 16 (right-side). 


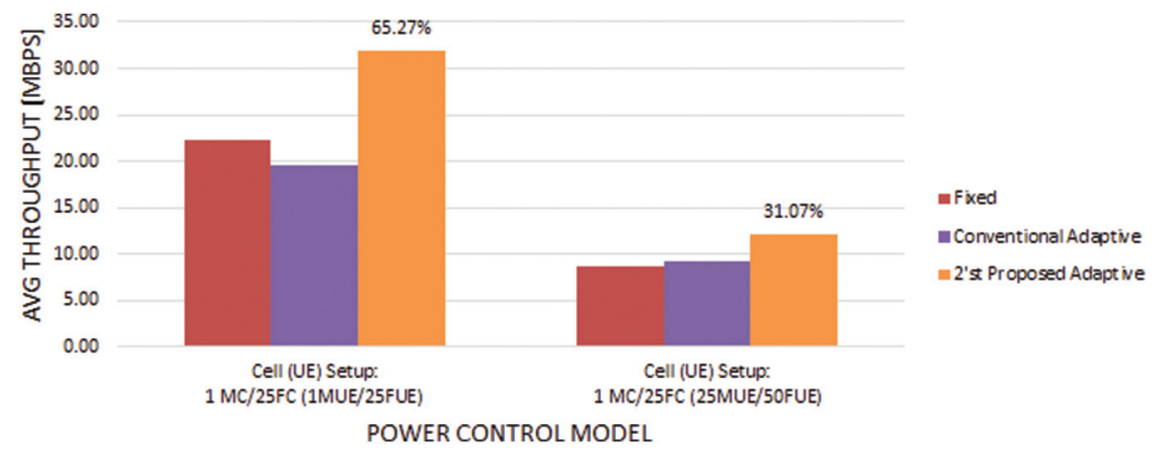

Figure 13 Average UE throughput in non-dense deployment.

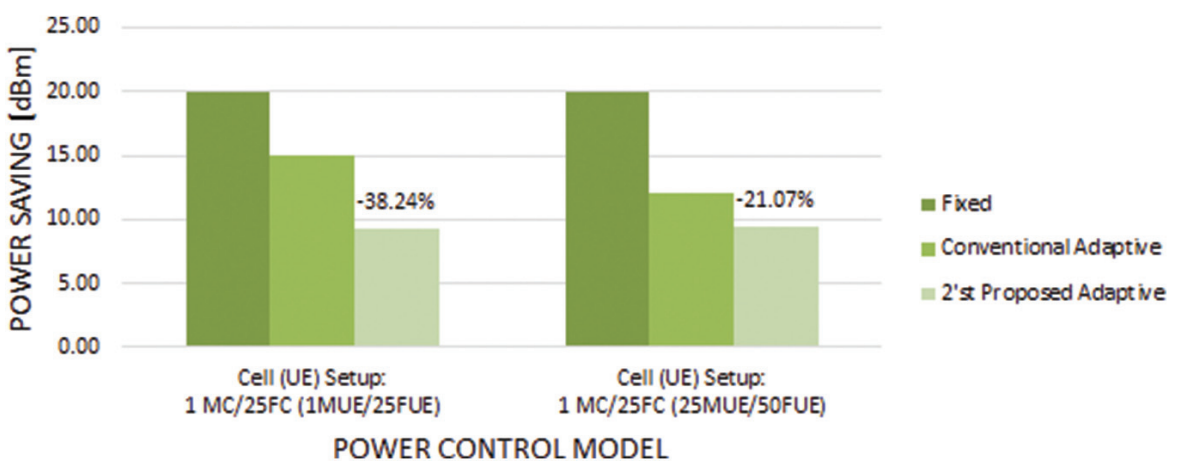

Figure 14 Power consumption per Femtocell in non-dense deployment.

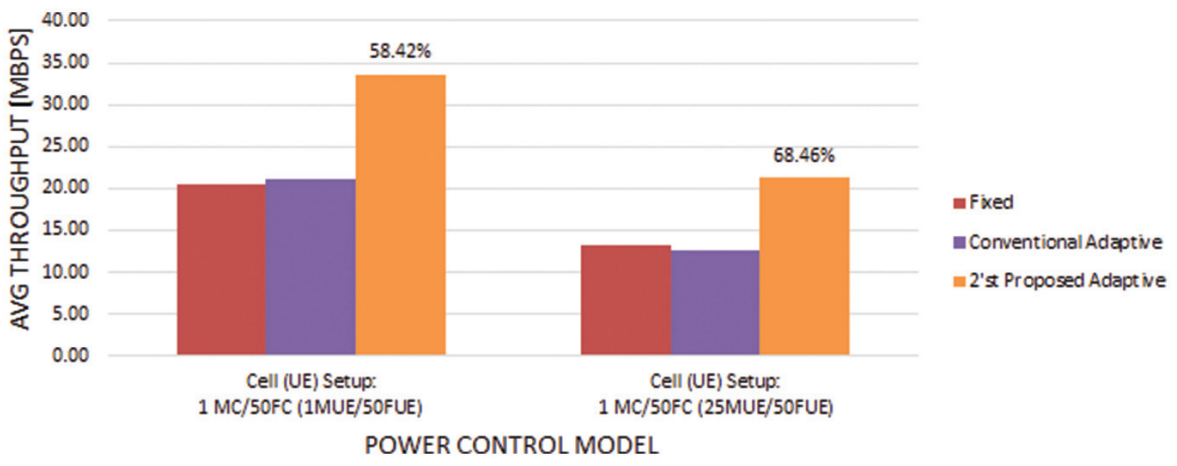

Figure 15 Average UE throughput in dense deployment. 
132 M. Al-Haddad and M. A. Bayoumi

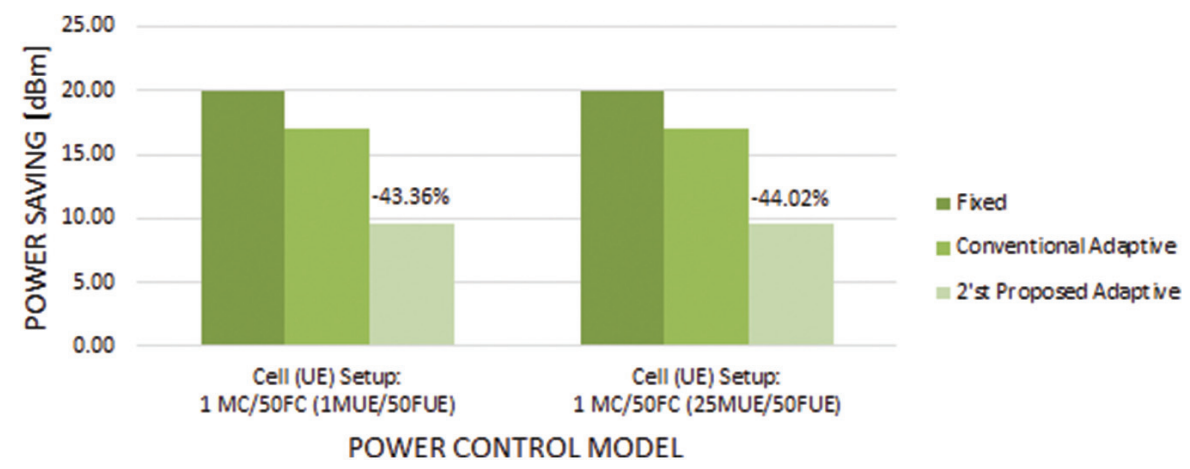

Figure 16 Power consumption per Femtocell in dense deployment.

With the introduction of our second new power control framework in Femtocell non-dense and dense deployments, we can see that the throughput has improved, a better power consumption has been achieved and the energy efficiency is higher. In comparison to our first proposed framework, where the measurement reports of attached Femtocell UEs are not considered, this Framework requires some additional signalling, as explained in Chapter 6, but provides more accurate results and higher power saving gain.

\subsection{Uplink Enriched FPC Based Framework}

For the sake of comparing the performance of our proposed framework for uplink power control versus recent related work, the following existing models have been considered:

1. Common OLPC, where $\mathrm{P}_{0}=-80 \mathrm{dBm}$ and alpha $=0.9$.

2. Different OLPC, where for Macrocell UEs we have $\mathrm{P}_{0}=-80 \mathrm{dBm}$ and $\alpha=0.9$, and for Femtocell UEs we have $\mathrm{P}_{0}=-59 \mathrm{dBm}, \alpha=0.6$ [42].

3. CLPC FPC, where $\alpha=0.8$ [26].

4. Adaptive power control, where $\mathrm{P}_{0}=\operatorname{round}\left(\mathrm{A}_{-} \mathrm{p}_{0}+\mathrm{Bp} 0 * \mathrm{PL}\right) \&$ for Macrocell UEs we have $\mathrm{P}_{0}=-80, \alpha=0.9$ and for Femtocell UEs we have $\mathrm{A}=-84 \mathrm{dBm}, \mathrm{B}=0.3, \alpha=0.6$ [21].

We consider two Macrocell clusters. One Macrocell, several buildings and $5 \times 5$ Femto cells per building are built in each cluster. Macrocell Cluster 1 is denser than cluster 2. In this way, we can check the dense and non-dense deployments. Simulated UEs (Femto and Macro related) are moving randomly with a speed of $3 \mathrm{~km} / \mathrm{h}$. The simulation results show the impact on UE uplink SIRN and UE power consumption in several deployment scenarios. 

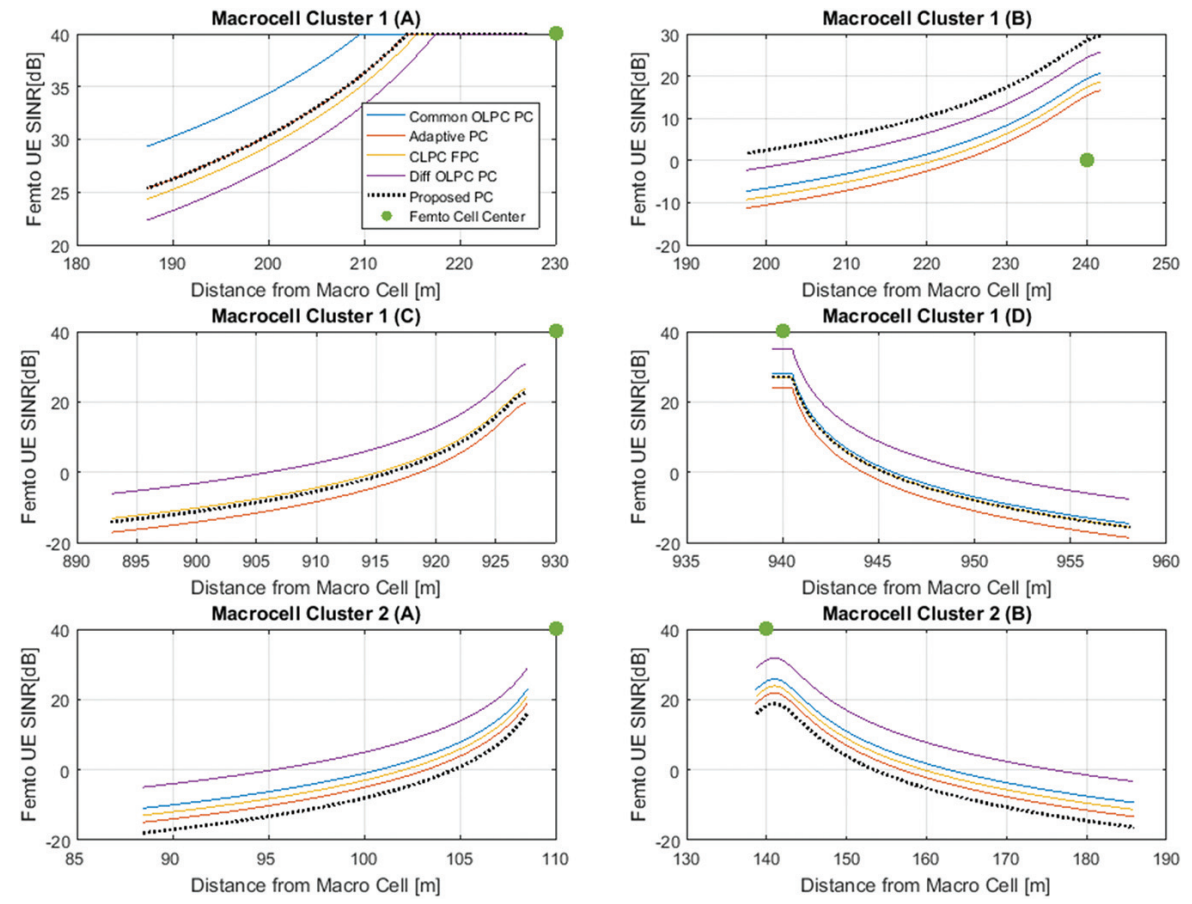

Figure 17 Femto UE SINR [dB].

Figure 17 shows the measured uplink SIRN per Femto UEs. Macrocell Cluster 1 (A, B, C and D), a massive deployment is simulated. The measured quality of Femto UE SINR [dB] is better than several other techniques. In Macrocell Cluster 2 (A and B), a light deployment is simulated. The measured quality of Femto UE SINR [dB] is acceptable and better than some other techniques. The setting of the SINR target is improved due to the way we identify Cell Interior and Edge UEs by taking into account the path loss to both serving and interfering cells, comparing to the other analyzed approaches based on PL to serving cell only.

Figure 18 shows the measured PUSCH per Femto UEs. In Macrocell Cluster 1 (A, B, C and D) and dense deployments, we can see a clear power saving for Edge and Interior UEs. In Macrocell Cluster 2 (A and B) where a non-dense environment is simulated, we can see Common OLPC is slightly better in terms of power consumed at the cell edge. However, our proposed algorithm is better in most of the simulated usecases. 

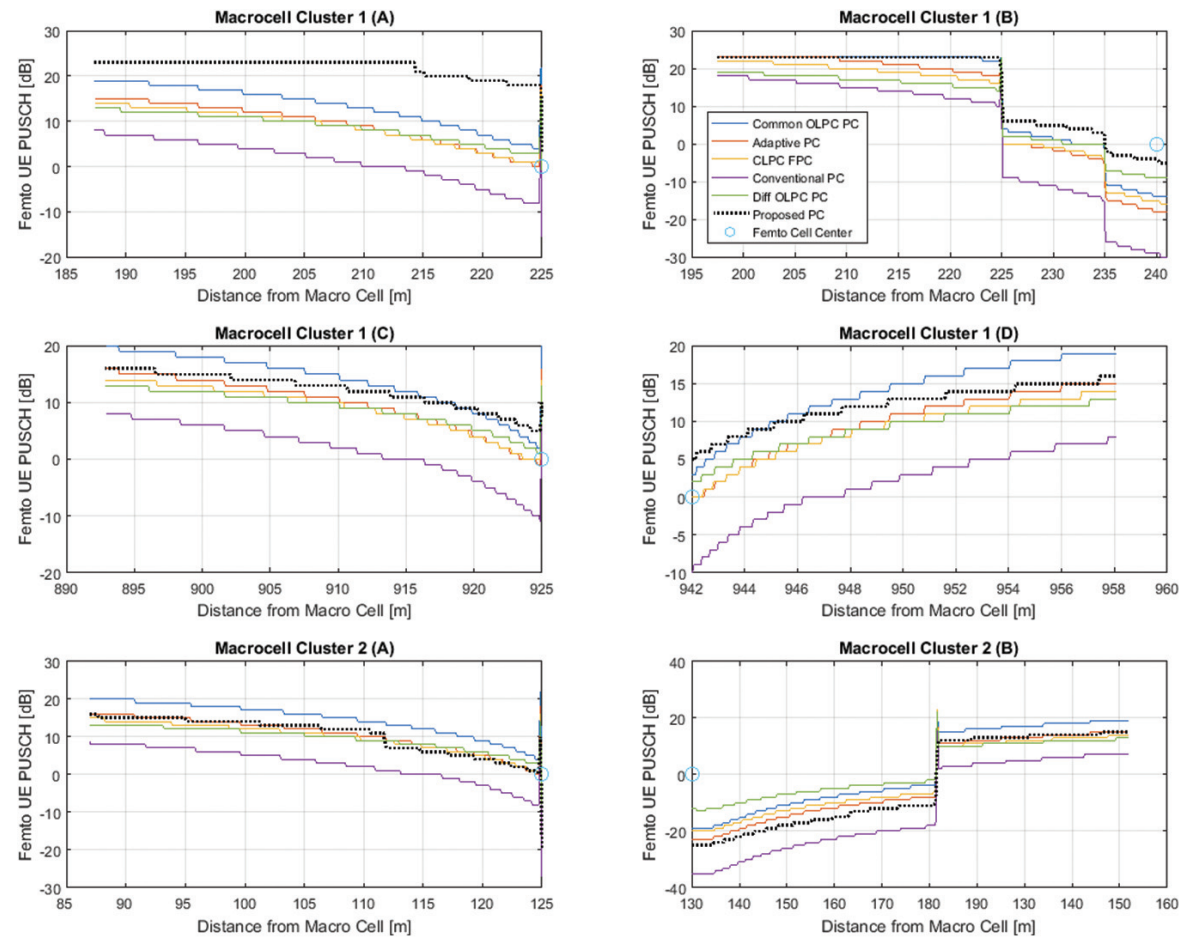

Figure 18 Femto UE PUSCH.

Figure 19 shows the measured uplink SIRN per Macro UEs. In cluster 1, the measured quality of Macro UE SINR [dB] is better than other techniques. Whereas in cluster 2 (non-dense deployment), it is equal to other techniques.

Figure 20 shows the measured PUSCH per Macro UEs.

In cluster 1 (dense deployment), some power saving is achieved for Edge UEs without losing quality. In this scenario up to $4 \mathrm{~dB}$ and $18 \%$ improvement between our proposed algorithm and the second best algorithm is achieved here. In the case of Interior UEs, some other algorithms have a slight jump. In cluster 2 (non-dense deployment), a clear power saving is achieved for both Edge and Interior UEs. Up to $20 \mathrm{~dB}$ saving and $90 \%$ improvement between our proposed algorithm and the second best algorithm has been measured.

All in all, the uplink transmission can work using standard UL power control methods. We showed it is possible to achieve gains from introducing Femtocells. However, achieving the gains requires very careful setting of UL PC parameters, where UEs connected to Femtocells transmit with lower power than Macro UEs. 

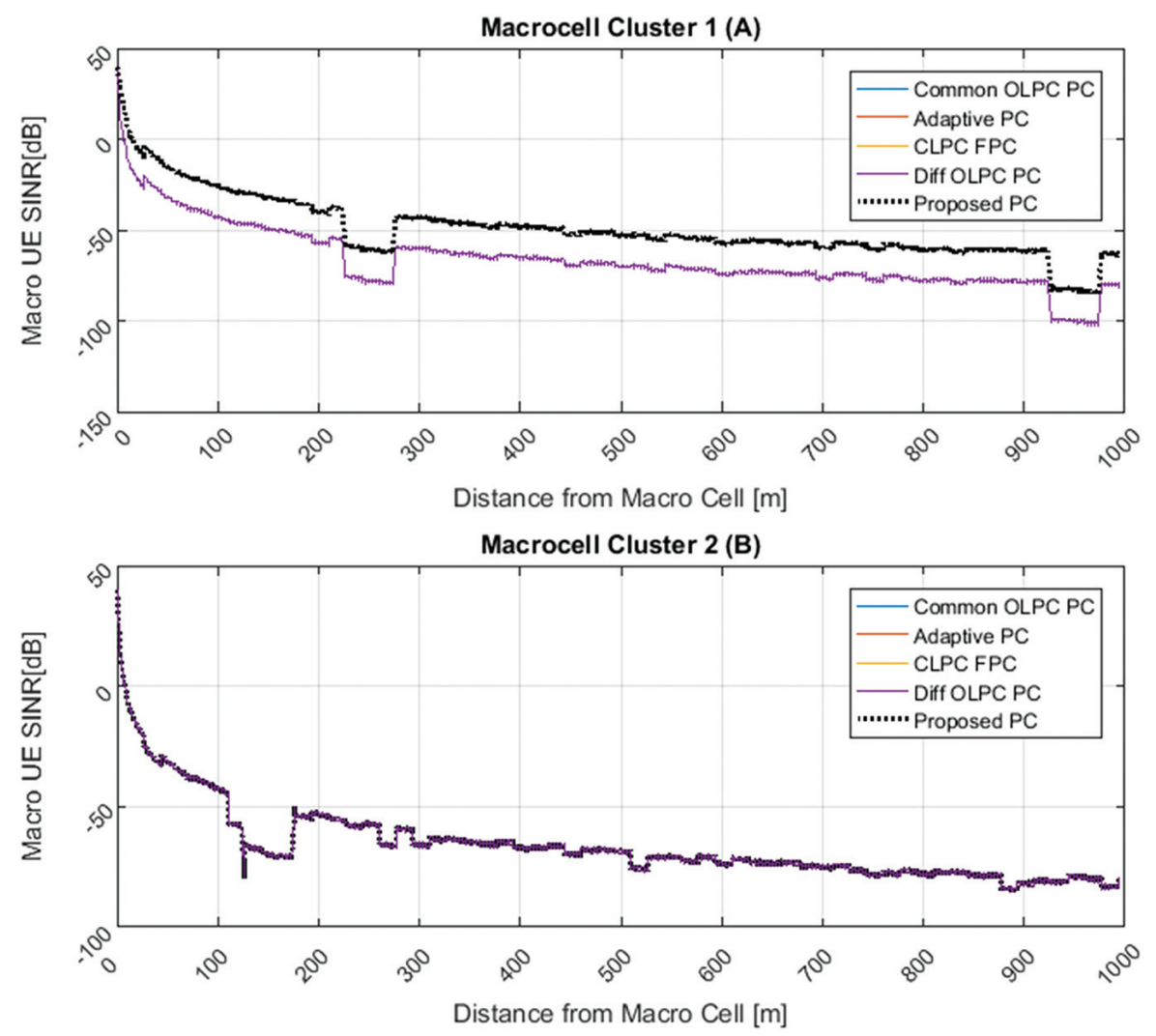

Figure 19 Macro UE SINR.

\subsubsection{Conventional vs. proposed fractional power control}

Figure 21 (left side) shows traditional fractional power control $(0<\alpha<1)$ where we plotted the result for different values of $\alpha$. SINR value is set to $15 \mathrm{~dB}$ and the number of PUSCH RB is 1 . The noise per RB considered is $-120.447 \mathrm{dBm}$. Figure 21 (right-side) shows the measured received PSD based on our proposed FPC, where densities of Femto \& Macro UEs are also considered (26 and 60). The PSD received varies depending on path loss and produces better curves.

In our proposed enhanced FPC, for lower path loss the user will transmit less power in comparison to existing conventional FPC power control methods. In the case of Cell Edge UEs, the target SNIR is set higher where the load situation of neighbouring Cells allows. 

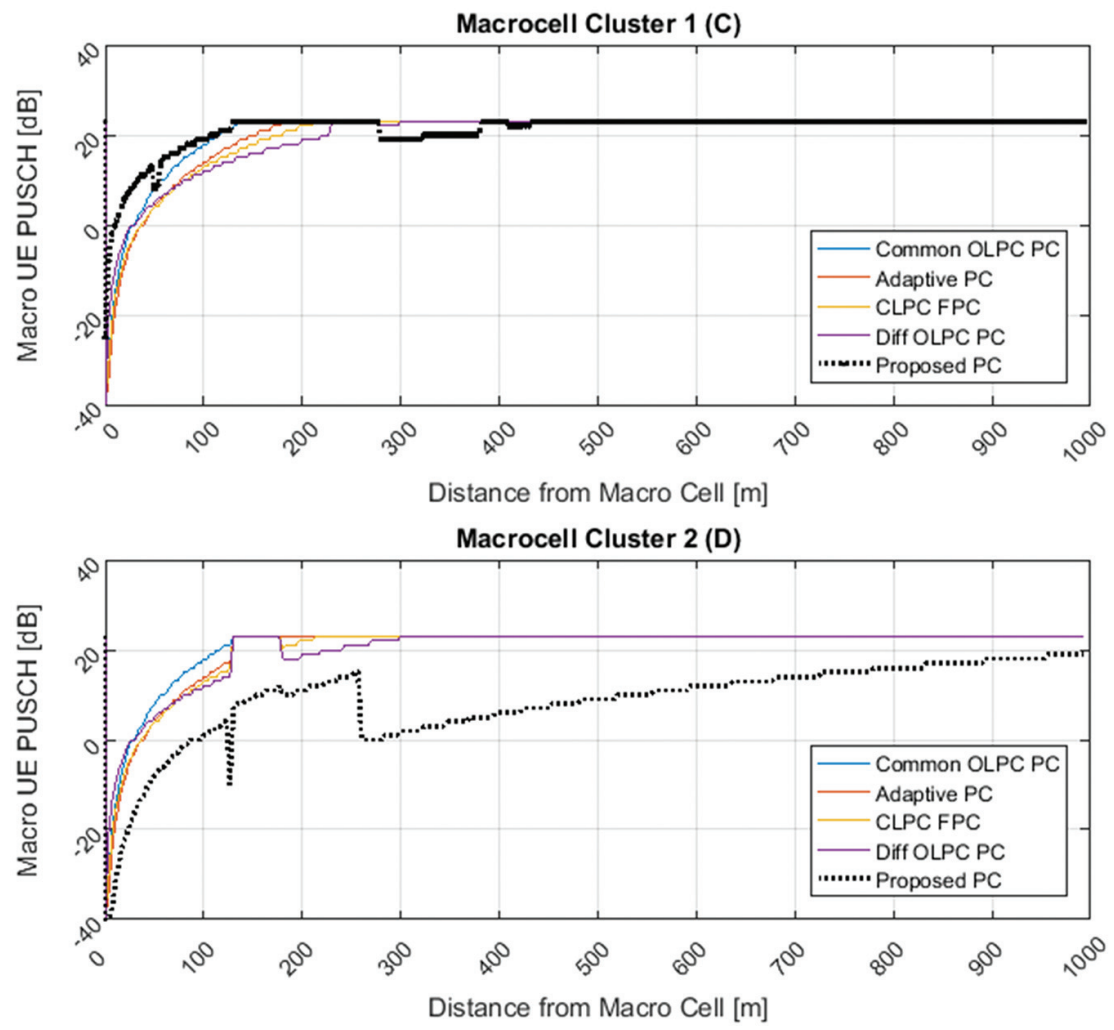

Figure 20 Macro UE PUSCH.
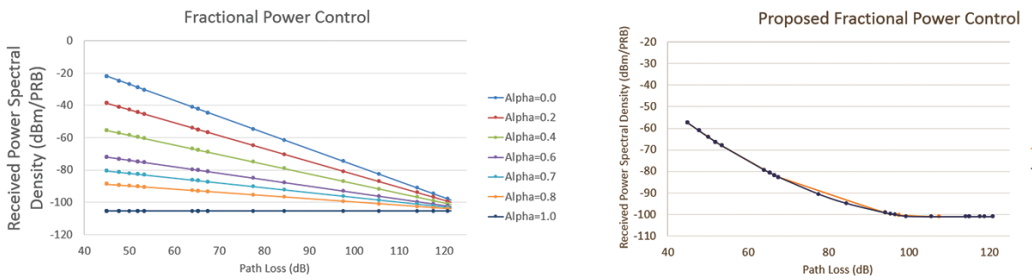

$\rightarrow$ Alpha=variable/UEs $=26$
$\rightarrow$ Alpha=variable/UEs $=60$

Figure 21 Conventional Fractional Power Control vs. Proposed one.

\subsubsection{Green impact \& $\mathrm{CO}_{2}$ emission}

Based on statistics reported in [41] for Base station power consumption (energy demand) at maximum load of a LTE system with $2 \times 2$ MIMO and the associated amount of $\mathrm{CO}_{2}$ estimated per year for one Femtocell in Italy reported in [40], the results of applying our power control proposed 
Table 3 Amount of $\mathrm{CO}_{2}$ emission saved per year (kg/year) for one Femtocell

\begin{tabular}{|c|c|c|c|c|}
\hline \multirow[b]{2}{*}{ Proposed Framework } & \multicolumn{2}{|c|}{ Dense Deployment } & \multicolumn{2}{|c|}{ Non-Dense Deployment } \\
\hline & $\begin{array}{l}\text { Max. Power } \\
\text { Saving (\%) }\end{array}$ & $\begin{array}{l}\mathrm{CO}_{2} \text { Saving } \\
\text { (kg/year)/Italy/ } \\
\text { Full Load Mode }\end{array}$ & $\begin{array}{l}\text { Max. Power } \\
\text { Saving (\%) }\end{array}$ & $\begin{array}{l}\mathrm{CO}_{2} \text { Saving } \\
\text { (kg/year)/Italy/ } \\
\text { Full Load Mode }\end{array}$ \\
\hline Downlink & 29.41 & 13.823 & 13.33 & 6.265 \\
\hline Distance-Based & & & & \\
\hline $\begin{array}{l}\text { Downlink } \\
\text { Measurement } \\
\text { Reports-Based }\end{array}$ & 44.02 & 20.689 & 38.24 & 17.973 \\
\hline Uplink Enriched FPC & 18.00 & 8.460 & 90.00 & 42.300 \\
\hline
\end{tabular}

Frameworks in Femtocell non-dense and dense deployments are calculated and shown in Table 3. Femtocell Full Load $\mathrm{CO}_{2}$ Emissions are assumed to be $47 \mathrm{~kg} /$ year and Femtocell Full Load Power Need is assumed to be $91.10 \mathrm{kWh} /$ year.

\section{Conclusion}

Femtocell technology is identified as a clear main 5G technology enabler. The gigantic growth in mobile data traffic and its associated energy consumption are having a significant impact on global warming and greenhouse gas emissions. In this research work, we have presented some novel and comprehensive frameworks for Femtocell power control in dense and nondense deployments to compensate for these effects and help in developing future-proof green networking solutions. Our objective is to make future Heterogeneous Networks energy-efficient and low $\mathrm{CO}_{2}$ emission consumers by developing more energy-aware radio access network technologies. Due to the ad-hoc nature and rapid proliferation of Femtocell networks and the presence of unpredictable environmental changes, our focus is more on distributed and self-organizing power control operations of Femtocells. A few approaches to mitigate interference and improve capacity in Femtocell Grids are discussed.

We have introduced two power control frameworks on Downlink which can greatly improve energy efficiency, coverage and quality of service. The first framework is based on distance and can mitigate the intra-interference across neighboring Femtocells in dense deployments, whereas the second one considers measurement reports from the attached UEs, Femto to Femto interference and the path loss of indoor Femto users. The second framework 
requires some additional signalling, but provides more accurate results and higher power saving. Both frameworks can be applied in different operational scenarios based on the Femtocell's ability to collect measurement reports from its UEs, hence the Femtocell should have a hybrid power setting mode and switch between the proposed frameworks as per need. We have also proposed one fractional power control framework for uplink where several types of interference, affecting parameters like Overload Indication status of neighbouring cells and RF changing circumstances are considered.

We used a system-level simulator for evaluating the impact of co-layer interference in indoor environment and the performance and power-saving gains achieved. The results show that our proposed frameworks can regulate Femtocell and UE power well and significantly reduce energy consumption, without degrading performance, by taking into account interference among Femtocells and many other surrounding RF circumstances. They demonstrated up to $44 \%$ power reduction and $68.46 \%$ higher UE throughput on downlink and up to $18 \%$ power reduction with $25 \%$ improved UE SINR on uplink in Femtocell dense deployments. This is a $\mathrm{CO}_{2}$ emission saving of up to 20.69 $\mathrm{kg} /$ year on downlink and $8.46 \mathrm{~kg} / \mathrm{year}$ on uplink for one Femtocell base station fed by a classical grid-powered solution. A comparison with the best-known power control modules is also covered in this study.

\section{References}

[1] Presentations by ABI Research, Picochip, Airvana, IP access, Gartner, Telefonica Espana, 2nd Intl. Conf. Home Access Points and Femtocells; available online at http://www.avrenevents.com/Accessed August 2012.

[2] Cisco, Visual Networking Index (VNI), 2014 (http://www.cisco.com/c/ en/us/solutions/collateral/service-provider/visual-networking-index-vni/ white_paper_c11-520862.html).

[3] Technology vision for the gigabit experience - Nokia networks 2014 (http://networks.nokia.com/file/26156/technology-vision-2020-whitepaper).

[4] "THE POWER OF WIRELESS CLOUD” An analysis of the energy consumption of wireless cloud-CEET Center for Energy-Efficient Telecommunications (Bell Labs and university of Melbourne).

[5] Bell Labs and university of Melbourne (2013), "THE POWER OF WIRELESS CLOUD" An analysis of the energy consumption of wireless cloud-CEET. 
[6] Mazen Al Haddad, Magdy Bayoumi "Evaluation of Femtocell Technology Challenges and Its Power Control Methodologies for Green Heterogeneous Networks"- SMARTGREENS 2014: Barcelona, Spain.

[7] Jo, H., Mun, C., Moon, J., Yook, J. Self-optimized coverage coordination in femtocell networks. IEEE Trans. Wirel. Commun. 9(10), 2977-2982 (2010).

[8] H. Leem, S.Y. Baek, D.K. Sung, The effects of cell size on energy saving, system capacity, and per-energy capacity, in: IEEE Wireless Communications and Networking Conference, Apr. 2010, pp. 1-6.

[9] Jing Huey Lim, R. Badlishah Ahmad, Muzammil Jusoh, Thennarasan Sabapathy "Power Management in LTE Femtocell Networks" - Springer International Publishing Switzerland 2015, Theory and Applications of Applied Electromagnetics Lecture Notes in Electrical Engineering Volume 344, 2015, pp 265-273, DOI 10.1007/978-3-319-17269-9_29.

[10] 3GPP TR 36.922: "Evolved Universal Terrestrial Radio Access (EUTRA); TDD Home eNode B (HeNB) Radio Frequency (RF) requirements analysis".

[11] Zhang, Haijun, Chu, Xiaoli, Wen, Xiangming "4G Femtocells Resource Allocation and Interference Management" SpringerBriefs in Computer Science 2013, DOT 10.1007/978-1-4614-9080-7, eBook ISBN 978-14614-9080-7.

[12] Xenakis, D.; Passas, N.; Merakos, L.; Verikoukis, C. "Mobility Management for Femtocells in LTE-Advanced: Key Aspects and Survey of Handover Decision Algorithms" - Communications Surveys \& Tutorials, IEEE (Volume: 16, Issue: 1).

[13] Jiming Chen ; Jimin Liu ; Peng Wang ; Jie Zhang "Backhaul ConstraintBased Cooperative Interference Management for In-Building Dense Femtocell Networks" - Vehicular Technology Conference (VTC Fall), 2012 IEEE.

[14] Lopez-Perez, D.; Ladanyi, A. ; Juttner, A. ; Jie Zhang "OFDMA Femtocells: A self-organizing approach for frequency assignment" - Personal, Indoor and Mobile Radio Communications, 2009 IEEE 20th International Symposium.

[15] RP - 111369, "Further enhanced non CA-based ICIC for LTE,"3GPP TSG RAN Meeting \#53, Fukuoka, Japan, 13-16 Sep. 2011.

[16] 3GPP R4-093349, "Femtocell and Macrocell interference coordination based on SFR," Motorola, RAN WG4 \#52, Aug., 2009.

[17] Shuguang Cui, Andrea J. Goldsmith, and Ahmad Bahai, "EnergyEfficiency of ?MIMO and Cooperative MIMO Techniques in Sensor 
Networks" IEEE Journal on Selected Areas in Communications, Vol. 22, No. 6, August 20041089.

[18] http://www.qualcomm.com/research/projects/lte-advanced/opportunisticsmall-cells

[19] 3GPP, "E-UTRA - Physical layer procedures", TS 36.213 v8.8.0

[20] 3GPPTS 36.104 Evolved Universal Terrestrial Radio Access (E-UTRA); Base Station (BS) radio transmission and reception; Release 9.

[21] Góra Jacek, Klaus I. Pedersen, Agnieszka Szufarska, Stanislaw Strzyz "Cell-Specific Uplink Power Control for Heterogeneous Networks in LTE” Vehicular Technology Conference Fall (VTC 2010-Fall), 2010 IEEE 72nd

[22] Vikram Chandrasekhar, Jeffrey G. Andrews, Tarik Muharemovic, Zukang Shen, Alan Gatherer "Power Control in Two-Tier Femtocell Networks" - IEEE Transactions on Wireless Communications, VOL. 8, NO. 8, AUGUST 2009.

[23] Ji-Hoon Yun and Kang G. Shin "CTRL: A Self-Organizing Femtocell Management Architecture for Co-Channel Deployment", MobiCom' 10, September 20-24, 2010, Chicago, Illinois, USA.

[24] Moushumi Sen $\dagger$, Suresh Kalyanasundaram $\dagger$, Rajeev Agrawal $\ddagger$ and Hans Kroener - "Performance Analysis of Interference Penalty Algorithm for LTE Uplink in Heterogeneous Networks" - 2014 IEEE.

[25] N. J. Quintero. Advanced Power Control for UTRAN LTE Uplink. Master's thesis, Aalborg University, 2008.

[26] B. Muhammad and A. Mohammed. Performance Evaluation of Uplink Closed Loop Power Control for LTE System. In Proc. of IEEE Vehicular Technology Conference VTC Fall, 2010.

[27] Marceau Coupechoux1, Jean-Marc Kelif „How to Set the Fractional Power Control Compensation Factor in LTE?" - Sarnoff Symposium, 2011 34th IEEE May 2011.

[28] Y. Zhang, Y. Yang, E. Sousa, and Q. Zhang, "Pilot Power Minimization in HSDPA Femtocells", in Proc. IEEE Global Communications Conference, Miami, USA, 2010, pp. 1-5.

[29] Sawsan A. Saad, Mahamod Ismail, and Rosdiadee Nordin "A Survey on Power Control Techniques in Femtocell Networks", in Journal of Communications Vol. 8, No. 12, December 2013.

[30] 3GPP R4-093557, "HeNB to Macro eNB Downlink Interference Mitigation with Power Control," NEC, RAN WG4 Meeting \#52bis, Oct., 2009. 
[31] Xiangfang Li, Lijun Qian, and Deepak Kataria, "Downlink Power Control in Co-Channel Macrocell Femtocell Overlay," Conference on Information Sciences and Systems (CISS), 2009, pp. 383-388.

[32] Motoki Morita, Yasuhiko Matsunaga, Kojiro Hamabe "Adaptive Power Level Setting of Femtocell Base Stations for Mitigating Interference with Macrocells" - Vehicular Technology Conference Fall (VTC 2010-Fall), 2010 IEEE 72nd

[33] Morita, M., Matsunaga, Y., Hamabe, K. "Auto-Tuning of Downlink Power of LTE Femtocells Adaptive to Various Interference Conditions" - Vehicular Technology Conference (VTC Spring), 2012 IEEE 75th

[34] 3GPP TR 36.902 - Evolved Universal Terrestrial Radio Access Network (E-UTRAN); Self-configuring and self-optimizing network (SON) use cases and solutions.

[35] Francesco Capozzi, Giuseppe Piro, Luigi Alfredo Grieco, Gennaro Boggia, and Pietro Camarda, "On accurate simulations of LTE Femtocells using an open source simulator", EURASIP Journal on Wireless Communications and Networking, 2012.

[36] 3GPP, Tech. Specif. Group Radio Access Network; Physical layer aspect for evolved Universal Terrestrial Radio Access (UTRA) (Release 7), 3GPP TR 25.814.

[37] 3GPP, R4-092042, Simulation assumptions and parameters for FDD HeNB RF requirements, 3GPP TSG RAN WG4 Meeting 51.

[38] IST-Winner II WINNER II Channel Models - Deliverable D1.1.2 V1.2 ([OnLine] Available: [http://www, 2007), ist-winner.org/WINNER2Deliverables/D1.1.2v1.2.pdf webcite].

[39] Al Haddad, Mazen, ElSayed, Zaghloul, Bayoumi, Magdy: Center for Advanced Computer Studies, University of Louisiana at Lafayette, USA, "Green arithmetic logic unit", 2012 International Conference on Energy Aware Computing (iceac).

[40] HetNets Powered by Renewable Energy: a Cost Effective Paradigm for Sustainable Next Generation Cellular Networks" 2013 IEEE Online Conference on Green Communications (IEEE Online GreenComm'13); $10 / 2013$.

[41] G. Auer et al., "How Much Energy Is Needed to Run a Wireless Network?" IEEE Wireless Comm., vol. 18, no. 5, 2011, pp. 40-49.

[42] 3GPP, "E-UTRA - Physical layer - Measurements", TS 36.214 v8.7.0. 


\section{Biographies}

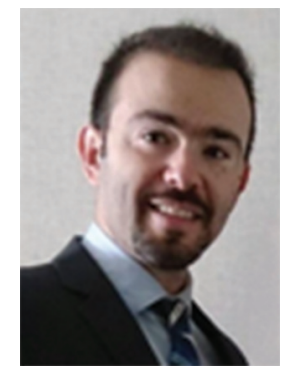

M. A. Haddad is a Ph.D. student at the University of Louisiana at Lafayette since spring 2011. He attended the University of Aleppo, Syria where he received his B.Sc. in Computer Engineering in 2002. Mazen then went on to purchase an M.Sc. in Computer Engineering from the University of DuisburgEssen, Germany in 2006. Mazen has held solution and software engineering senior positions at Nokia Networks since 2004. He, as a Telecommunication expert and professional, has acquired a solid experience in service quality management of Telecommunication networks. Mazen is currently completing a doctorate in Computer Science at the University of Louisiana at Lafayette. His Ph.D. work centers on Green Telecommunication and discusses the green networking movement to develop new energy efficient and green solutions.

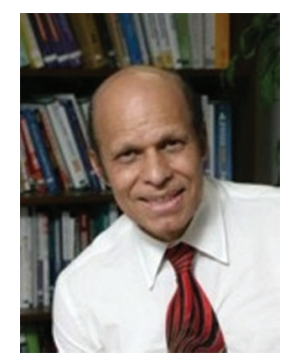

M.A. Bayoumi received his B.Sc. and M.Sc. degrees in Electrical Engineering from Cairo University, Egypt; M.Sc. degree in Computer Engineering from Washington University, St. Louis; and Ph.D. degree in Electrical Engineering from the University of Windsor, Canada. Dr. Bayoumi is the recipient of the 2009 IEEE Circuits and Systems Meritorious Service Award. Dr. Bayoumi is the recipient of the IEEE Circuits and Systems Society 2003 Education Award, and he is an IEEE Fellow. He was on the governor's commission 
for developing a comprehensive energy policy for the State of Louisiana. He represented the CAS Society on the IEEE National Committee on Engineering R\&D policy, IEEE National Committee on Communication and Information Policy, and IEEE National Committee on Energy Policy. He is also active in the "Renewable \& Green Energy" and "Globalization: Technology, Economic and Culture" fields. He was a free-lance columnist for Lafayette's newspaper. He is also the Z.L. "Zeke" Loflin Eminent Scholar Endowed Chair at the Center for Advanced Computer Studies, University of Louisiana, Lafayette. Dr. Bayoumi has been a faculty member in CACS since 1985 and has graduated more than 44 Ph.D. and about 175 Master's students. He has published over 300 papers in related journals and conferences. He edited, co-edited and co-authored 5 books in his research interests. He was and has been Guest Editor (or Co-Guest Editor) of eight special issues in VLSI Signal Processing, Learning on Silicon, Multimedia Architecture, Digital and Computational Video, Perception on a Chip, and Systems on a Chip. He has given numerous invited lectures and talks nationally and internationally, and has consulted in industry. His research interests include VLSI design methods and architectures, low power circuits and systems, digital signal processing architectures, parallel algorithm design, computer arithmetic, image and video signal processing, neural networks, and wideband network architectures. 
